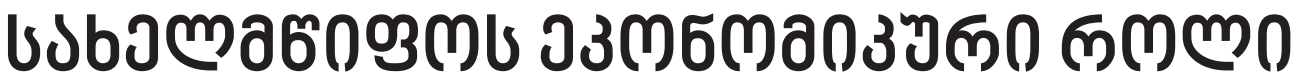

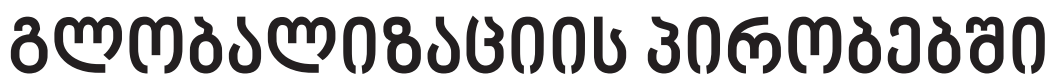

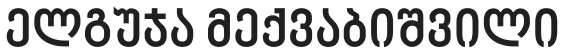

эзмбмдаз з

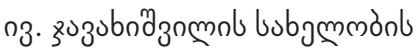

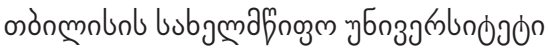

bufurnos zammml azmbmanz

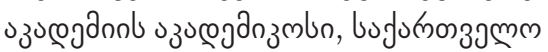

elguja.mekvabishvili@tsu.ge

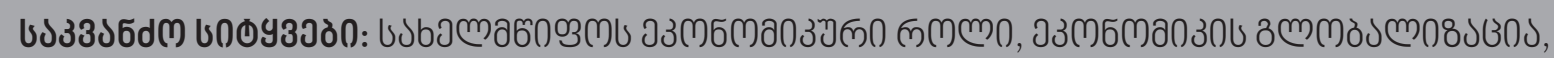

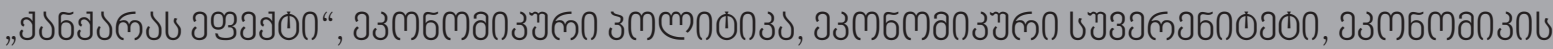

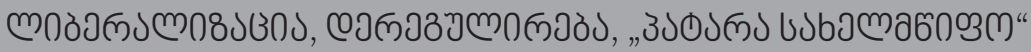

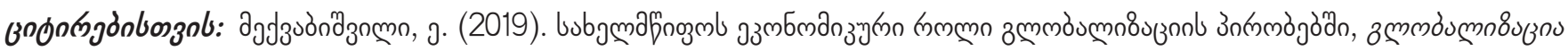
cou òn65jlo, №8, 33· 22-33. https://doi.org/10.35945/gb.2019.08.002

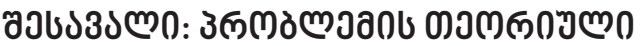

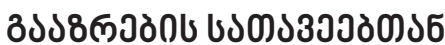

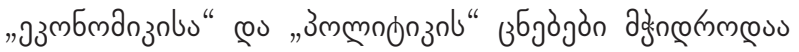

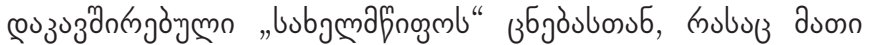

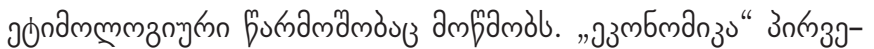

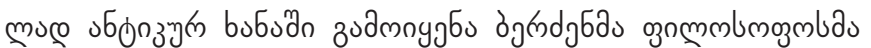

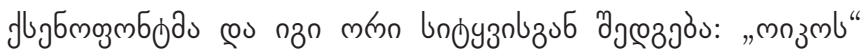

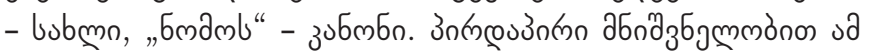

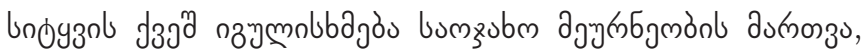
zudmmgno.

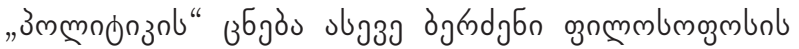

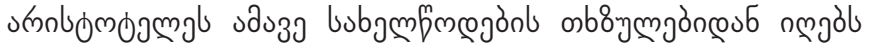

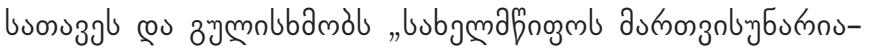

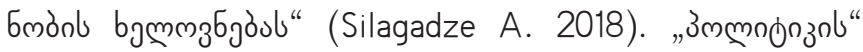

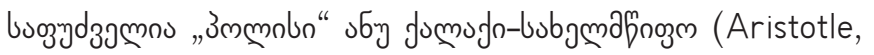

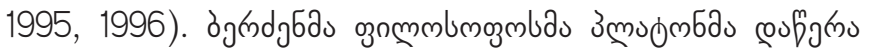

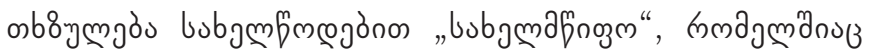

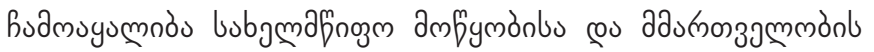

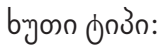

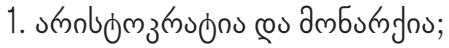

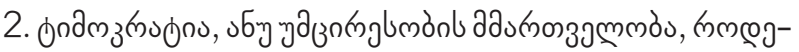

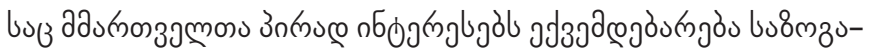

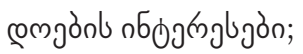

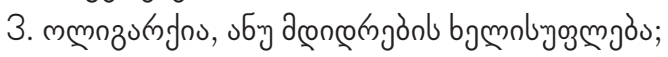

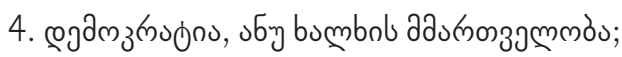

5. onkubos.

u

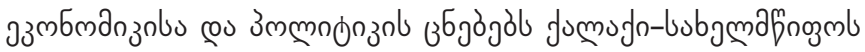

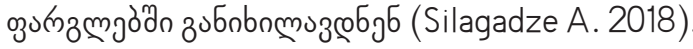

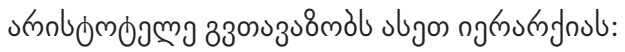

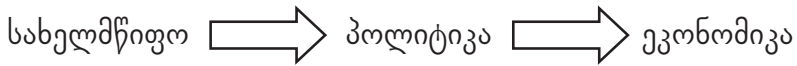

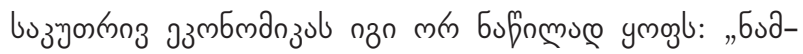

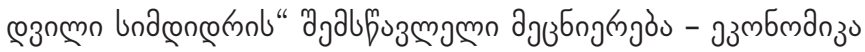

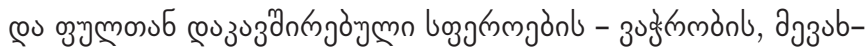

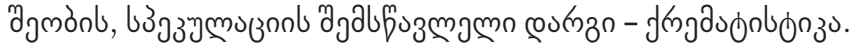

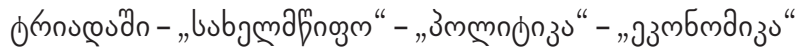

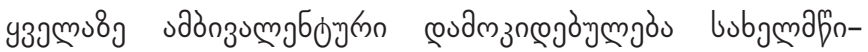

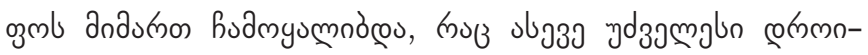

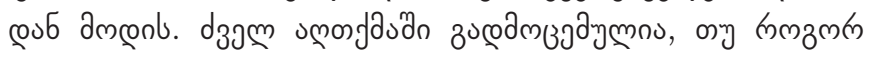

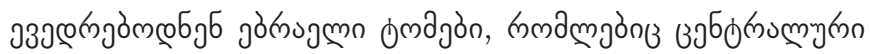

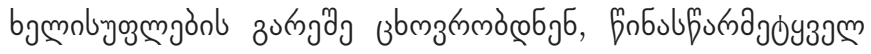

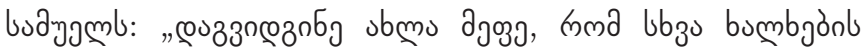

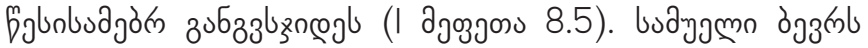

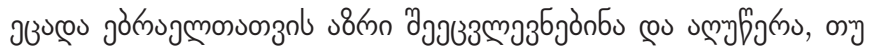

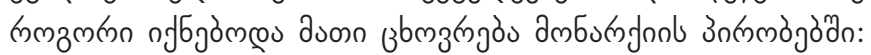

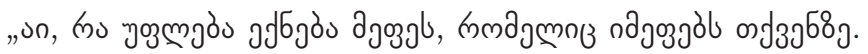

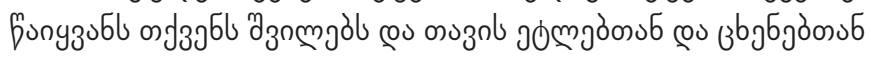




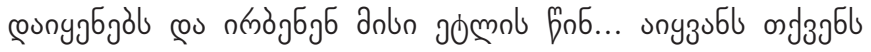

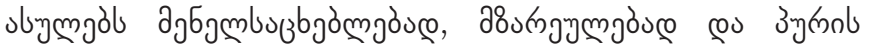

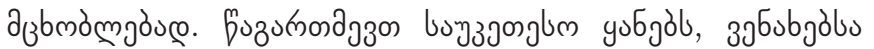

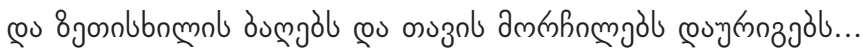

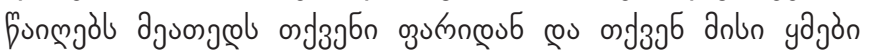

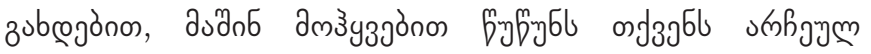

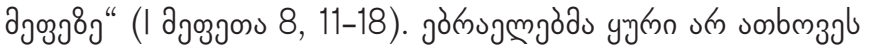
fobulfusmagogzamol dazmbjoul (Roseanne H. S. 2006) po

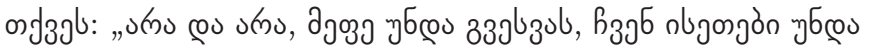

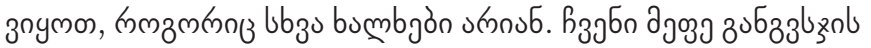

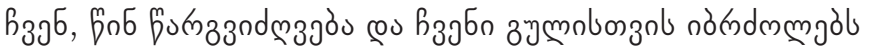

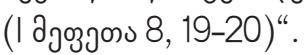

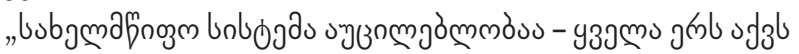

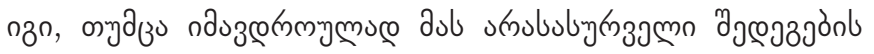
amoubusz gagdmos" (Roseanne H. S. 2006).

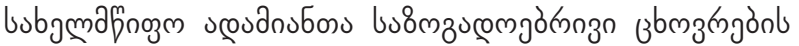

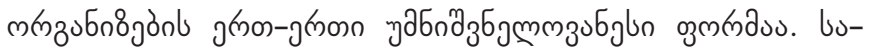

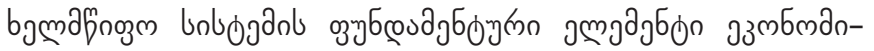

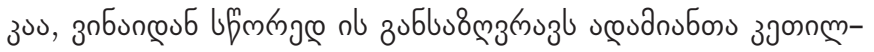

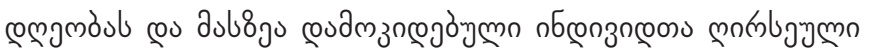

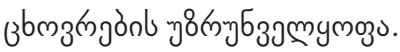

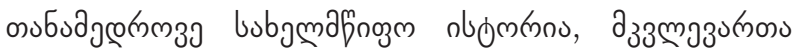

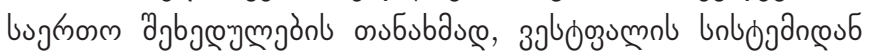

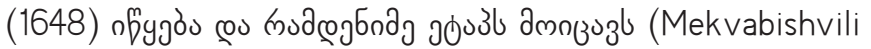

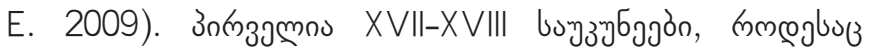

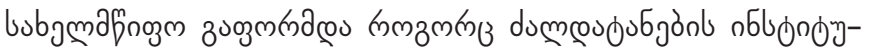

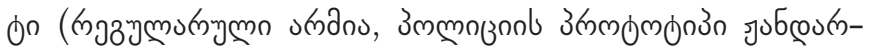

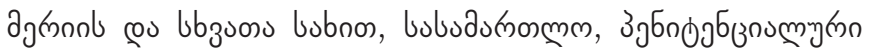

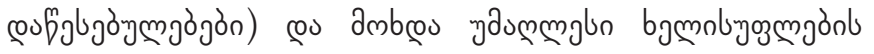

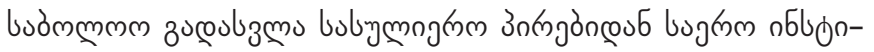

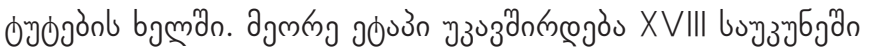

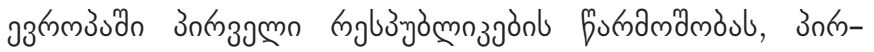

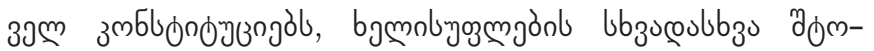

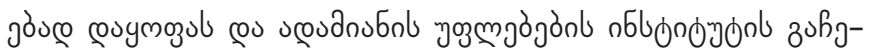

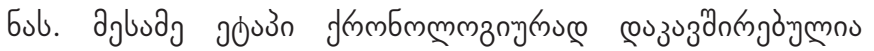

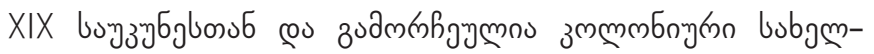

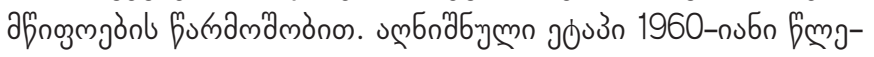

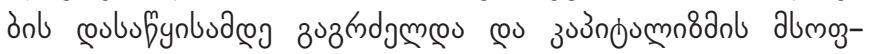

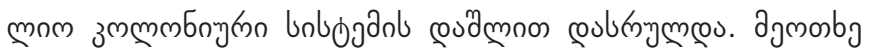

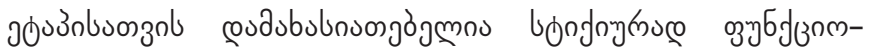

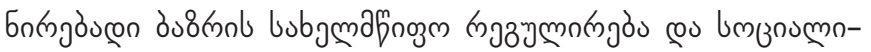

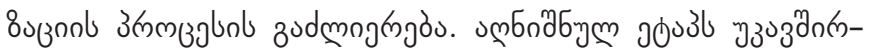

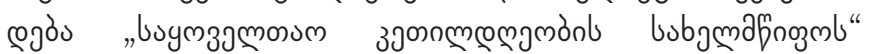

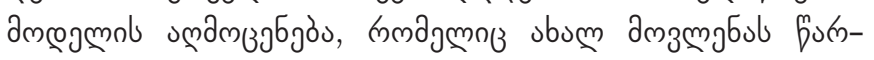

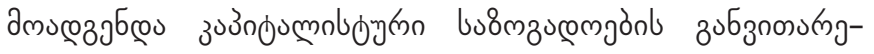

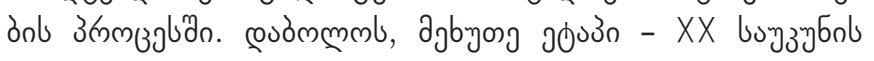

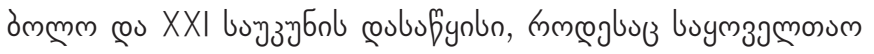

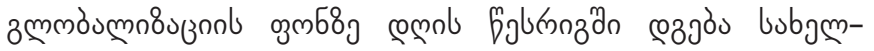

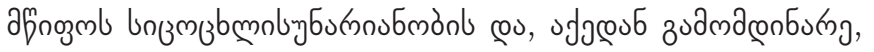

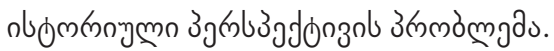

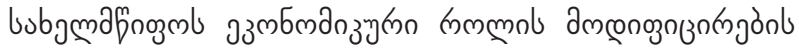

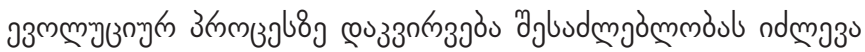

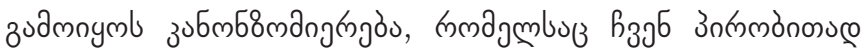

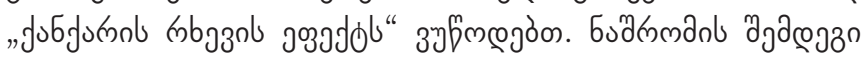
उง muzgruogn uf

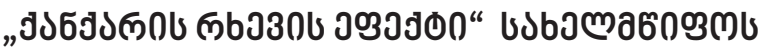

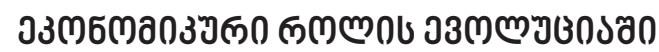

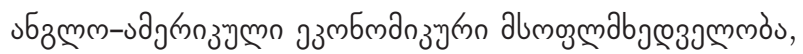

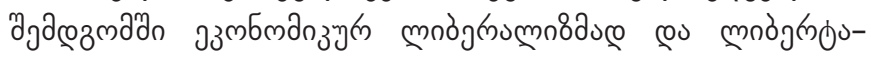

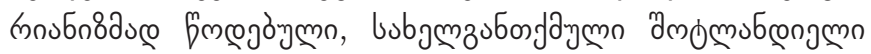

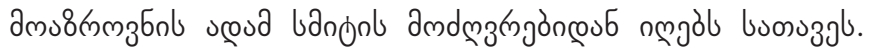

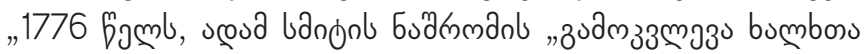

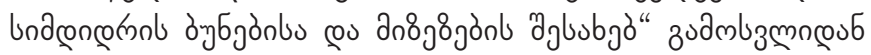

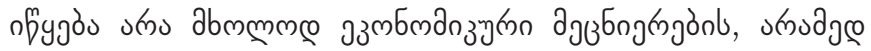

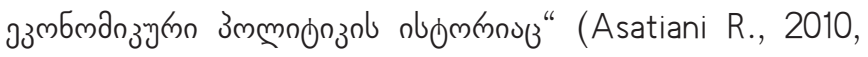
$33.11)$.

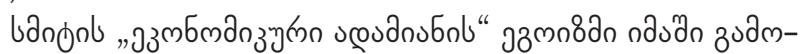

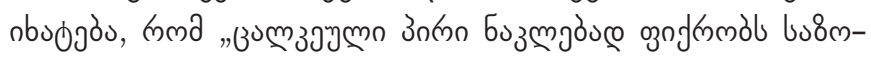

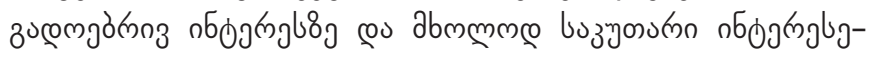

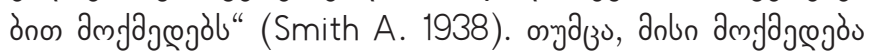

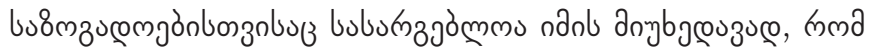

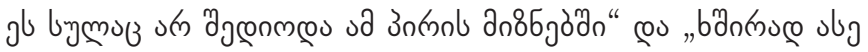

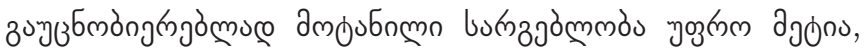

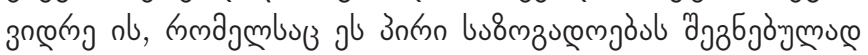

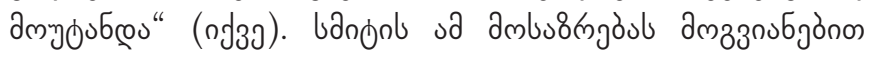

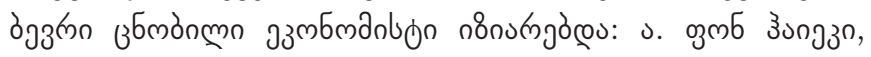

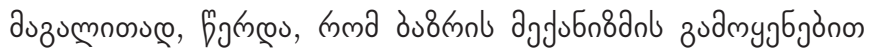

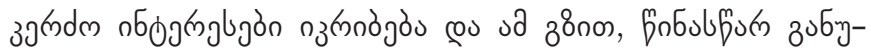

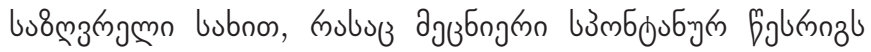

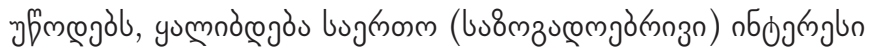

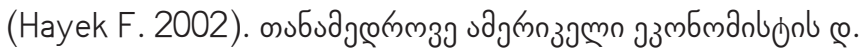

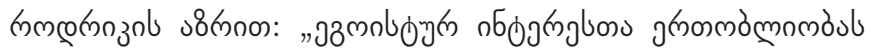

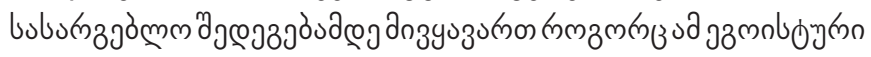

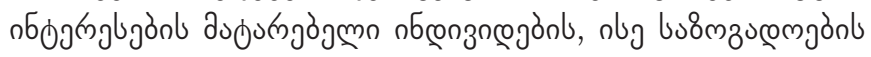

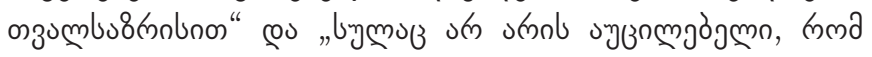

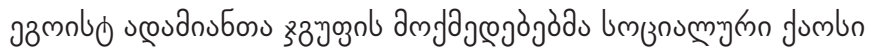
zudmofзлmb" (Rodrik D. 2017).

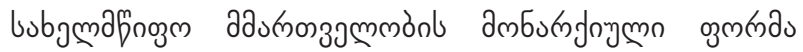

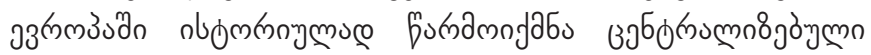

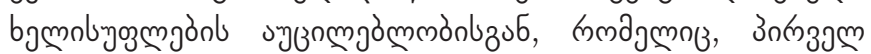

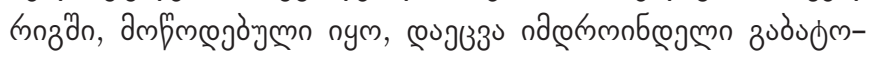

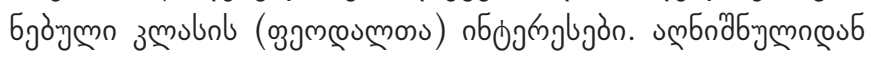

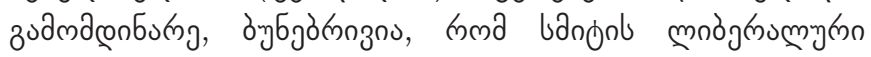

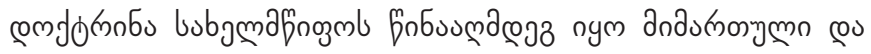

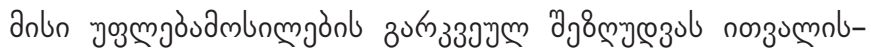

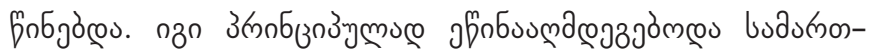

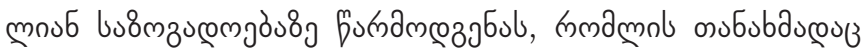




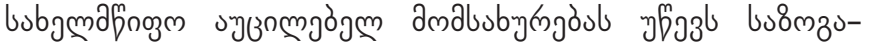

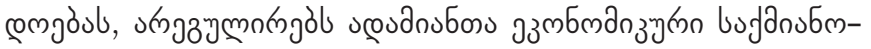

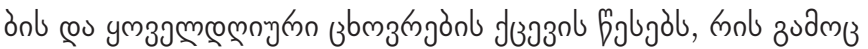

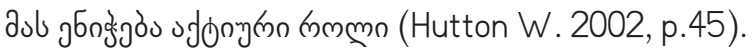

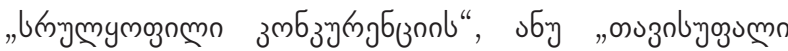

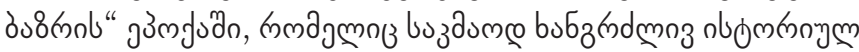

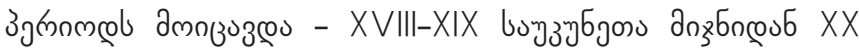

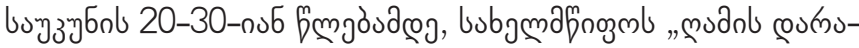

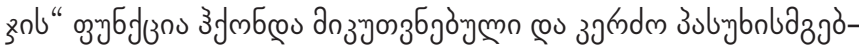

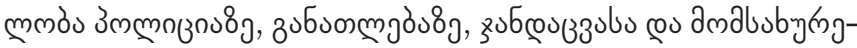

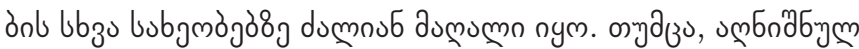

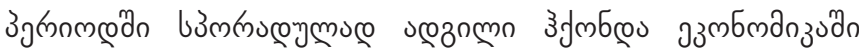

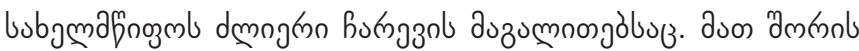

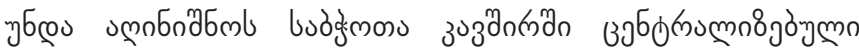

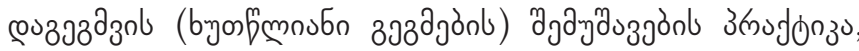

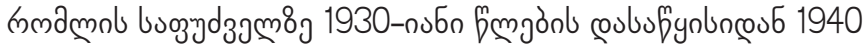

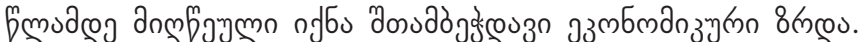
3j

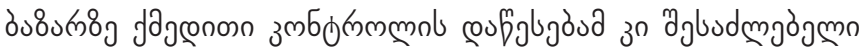

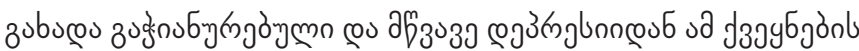

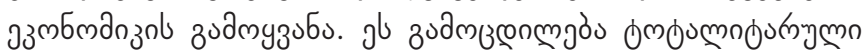

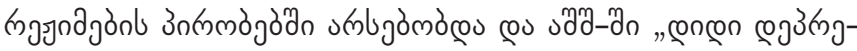

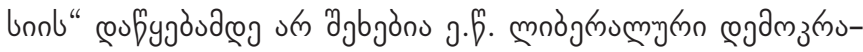
jonb bubgmafnnogmabl.

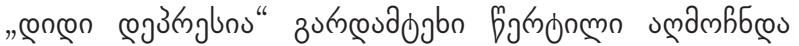

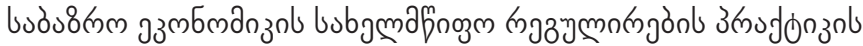

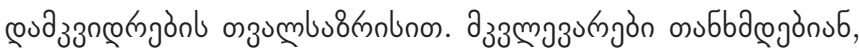

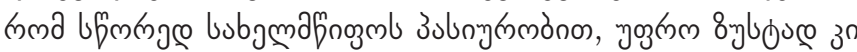

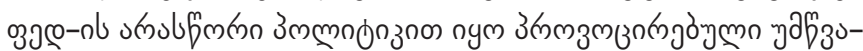

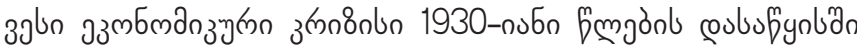

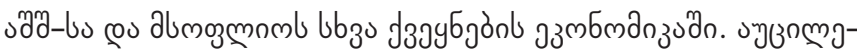

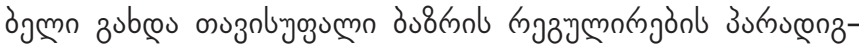

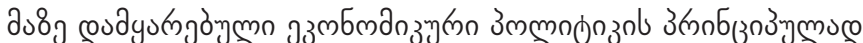

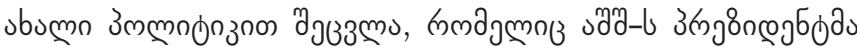

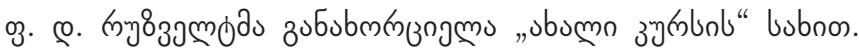

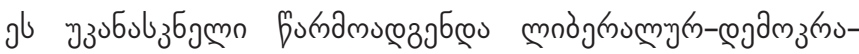

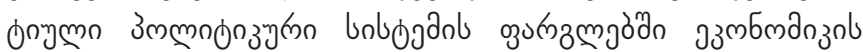

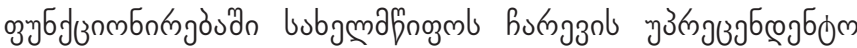

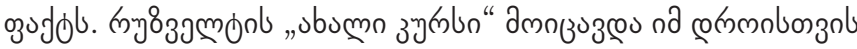

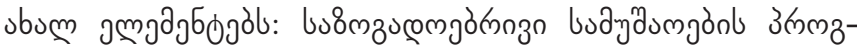

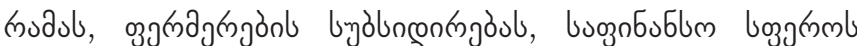

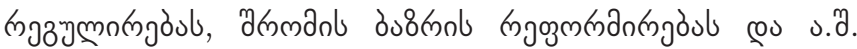

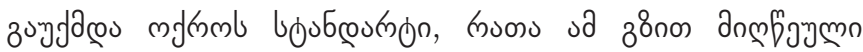

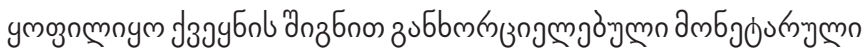

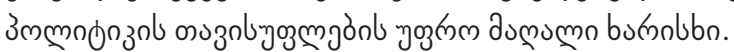

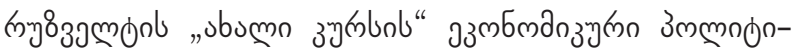

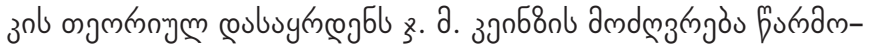

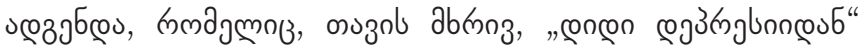

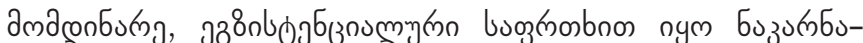

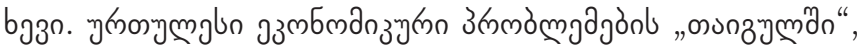

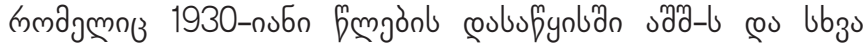

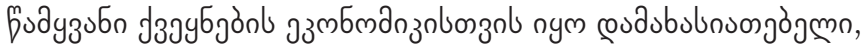

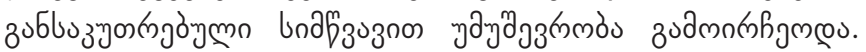

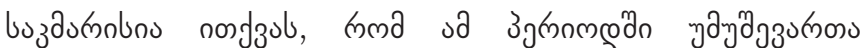

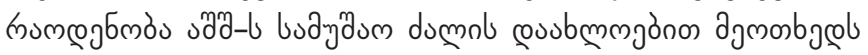

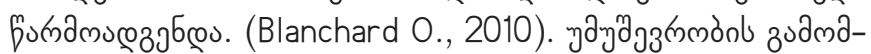

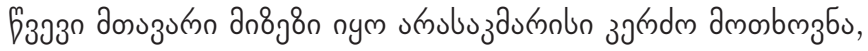

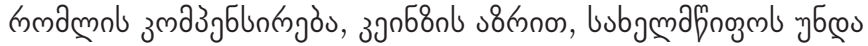

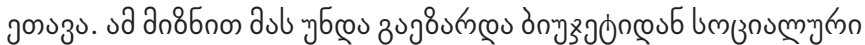

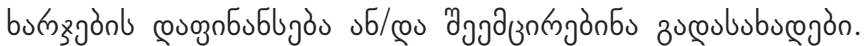

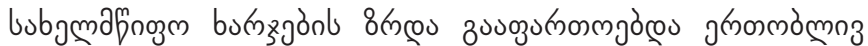

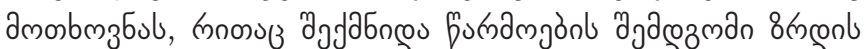

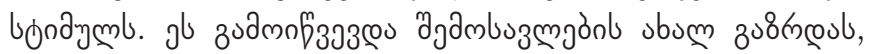

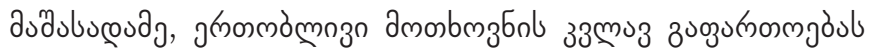

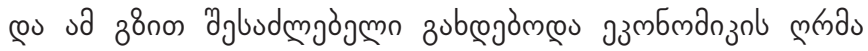
z mo8onbncoub zuamyzubs.

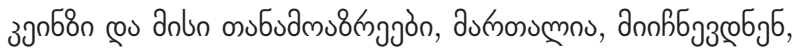

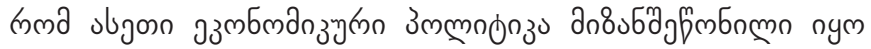

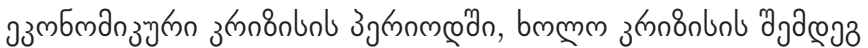

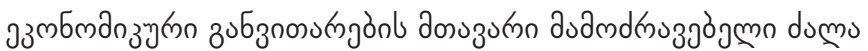
n

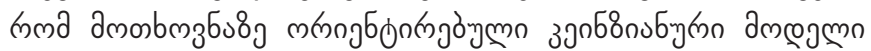

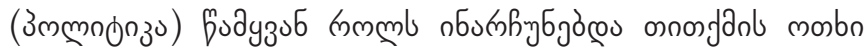

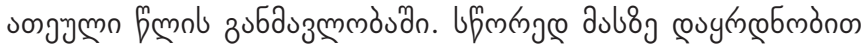

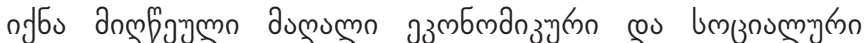

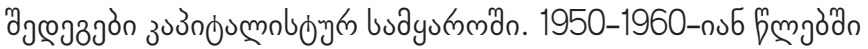

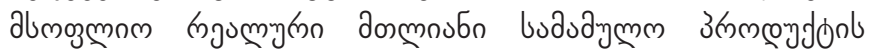

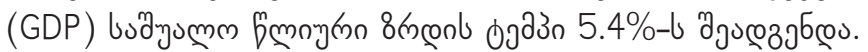

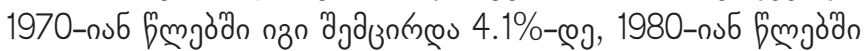

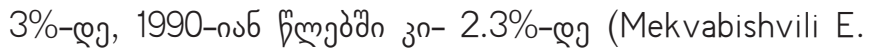

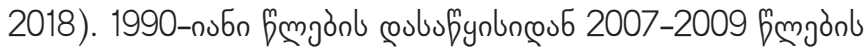

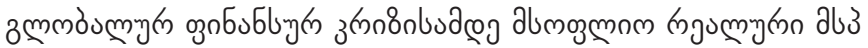

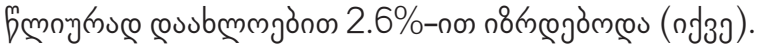

2бпдзб

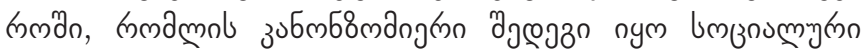

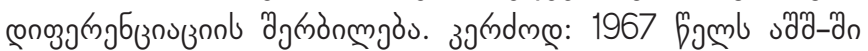

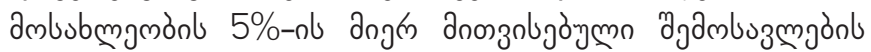

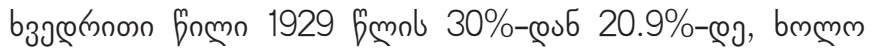

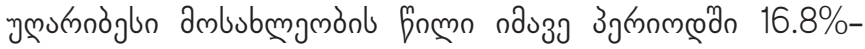

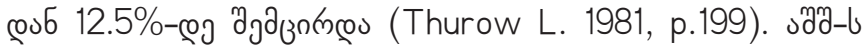

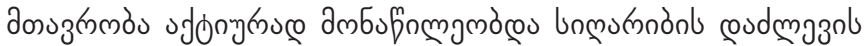

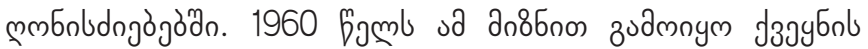

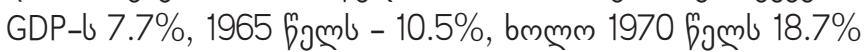
(Pierson Ch., 1995, p.128).

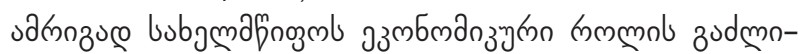

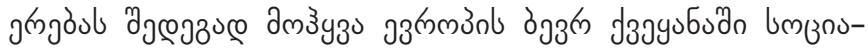

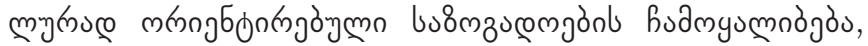

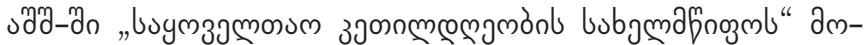

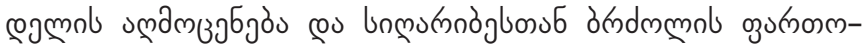

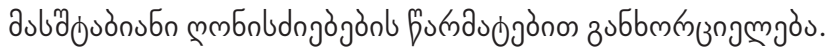




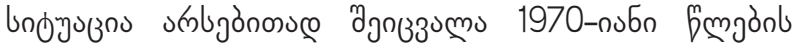

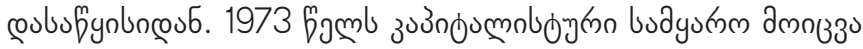

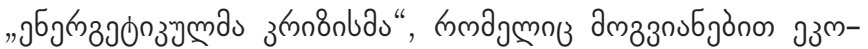

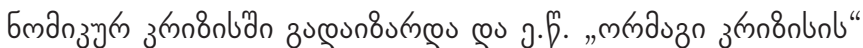

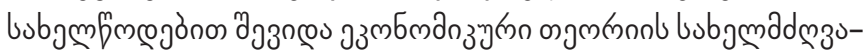

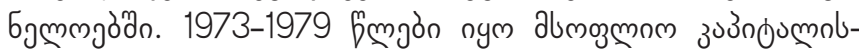

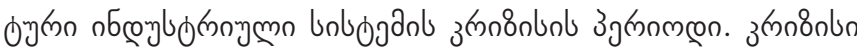

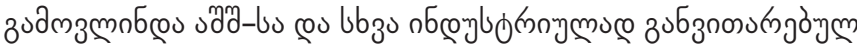

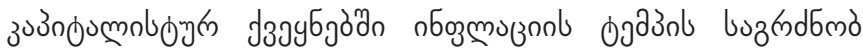

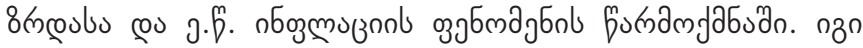

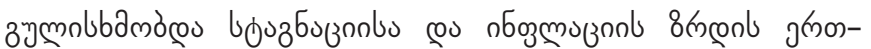

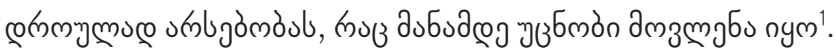

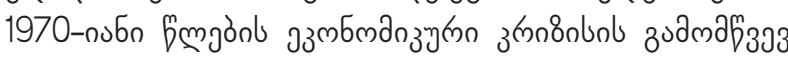

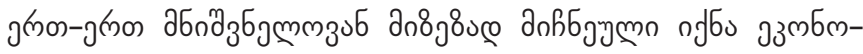

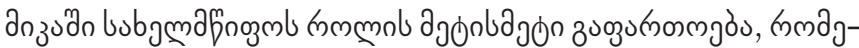

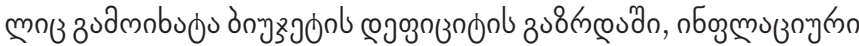

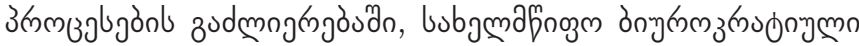

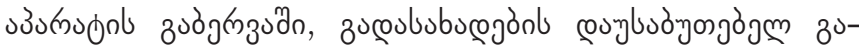
8m

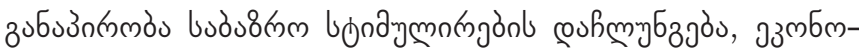

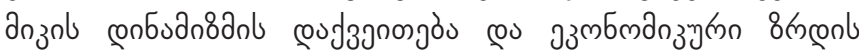

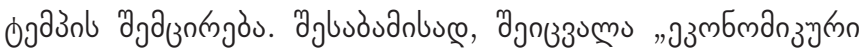

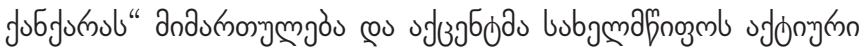

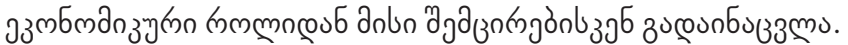

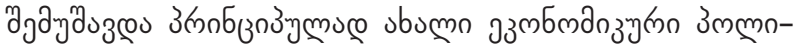

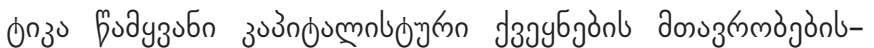

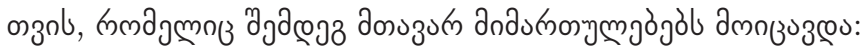

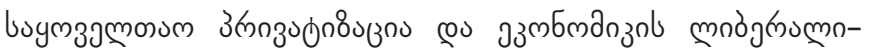

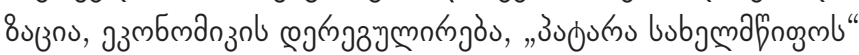

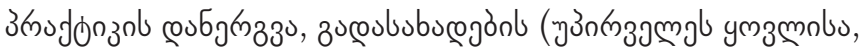

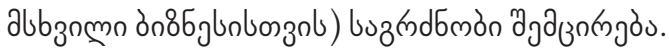

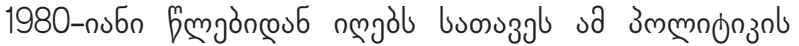

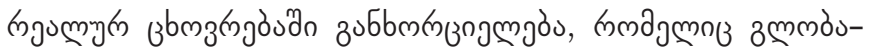

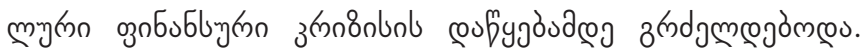

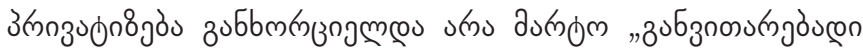

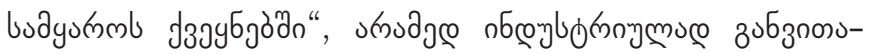

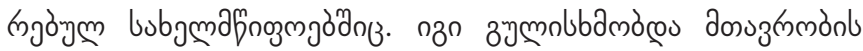

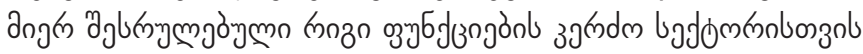

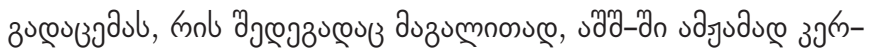

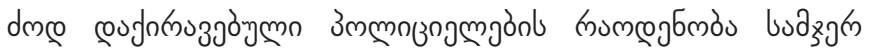

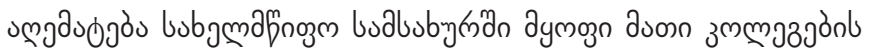

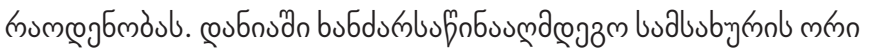

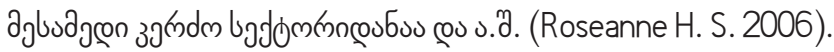

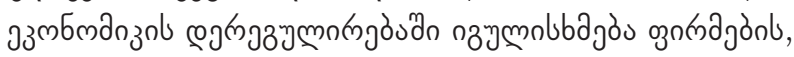

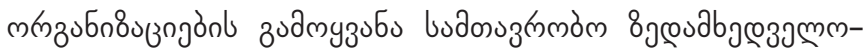

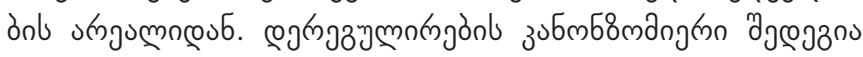

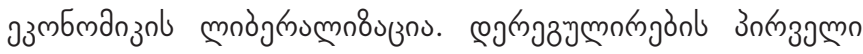

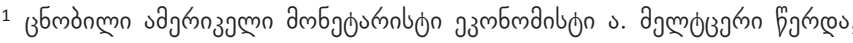

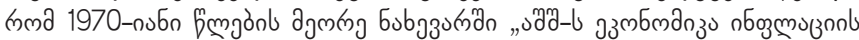

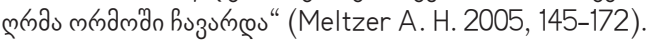

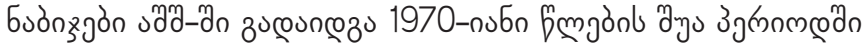

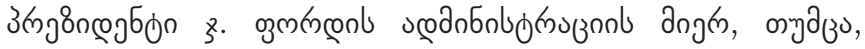

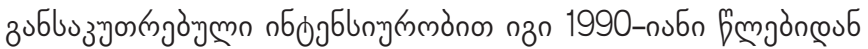

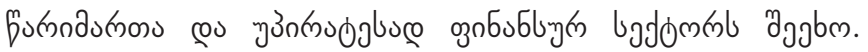

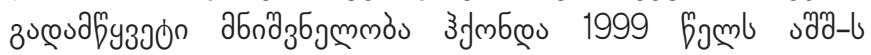

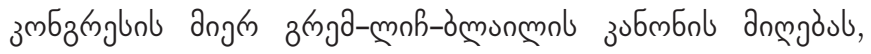

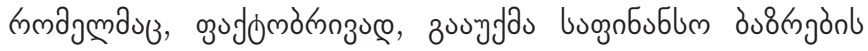

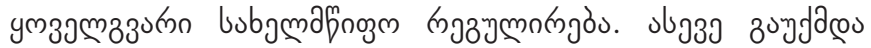

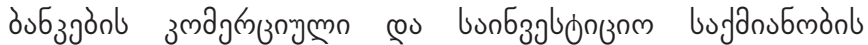

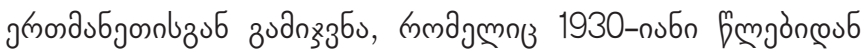

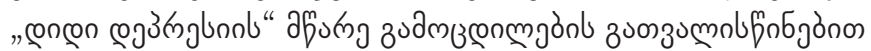

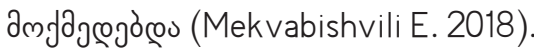

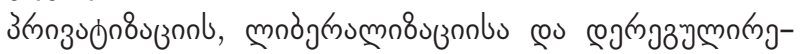

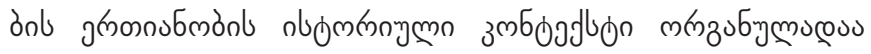

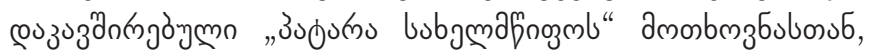

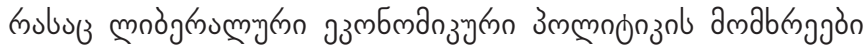

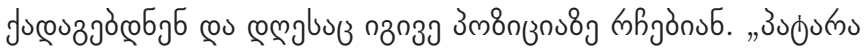

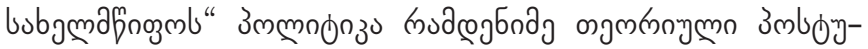

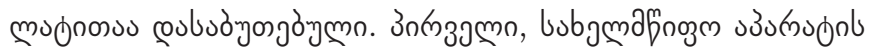

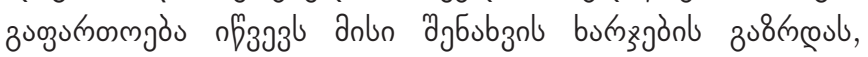

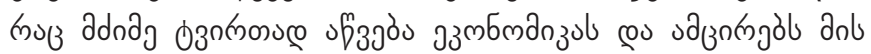

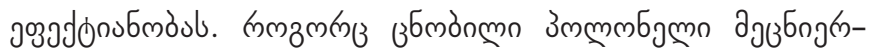

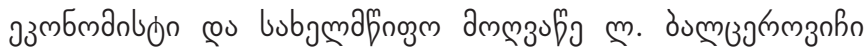

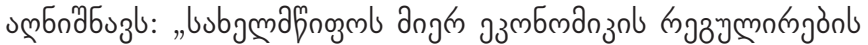

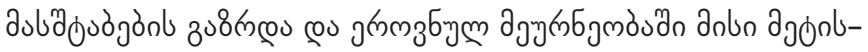
ajon nбол

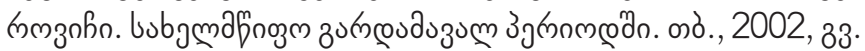

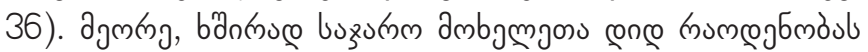

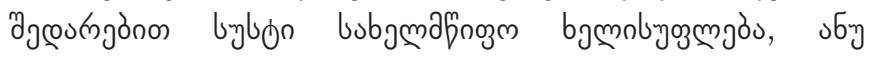

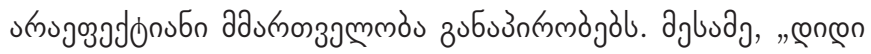

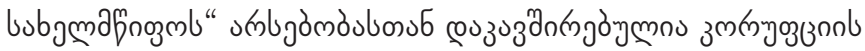

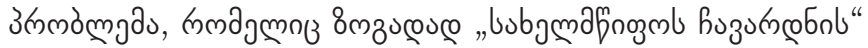

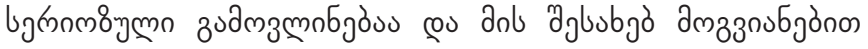

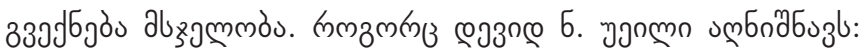

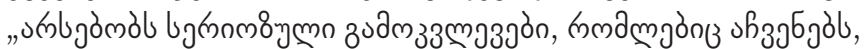

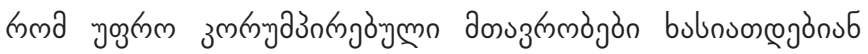

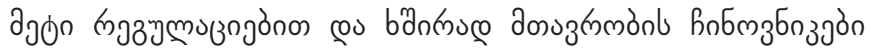

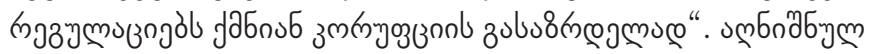

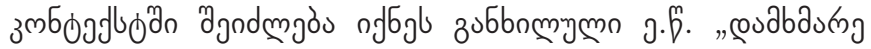

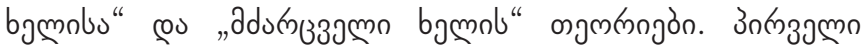

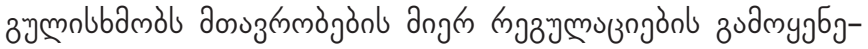

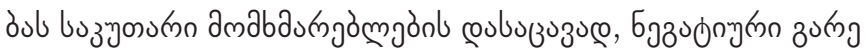

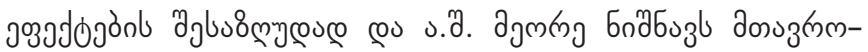

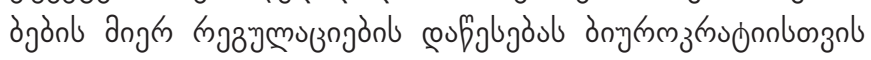

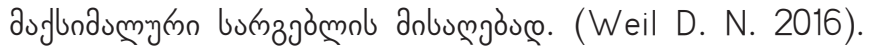

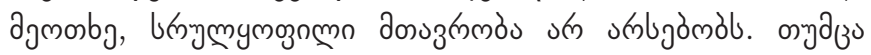

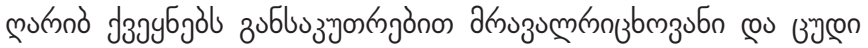

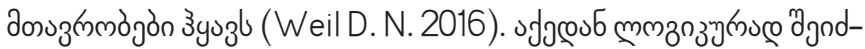

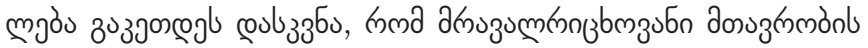

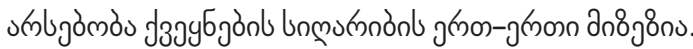




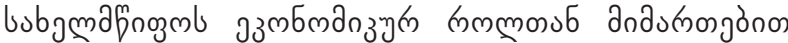

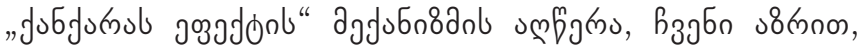

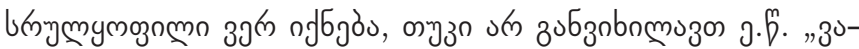

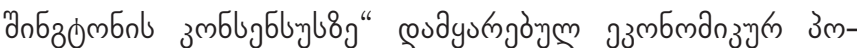

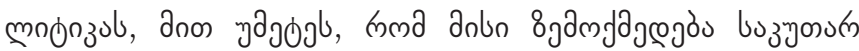

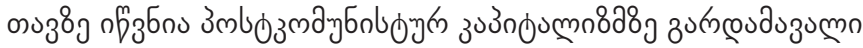

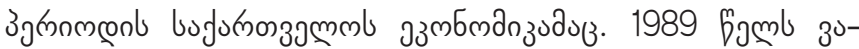

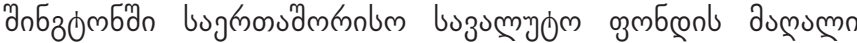

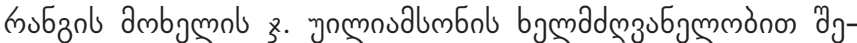

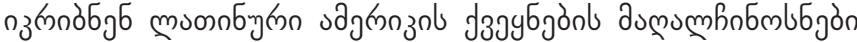

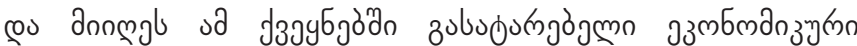

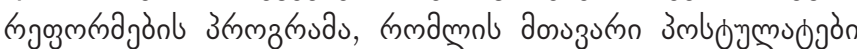

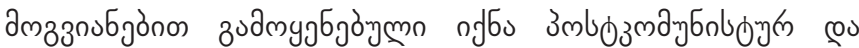

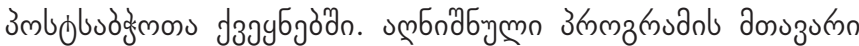

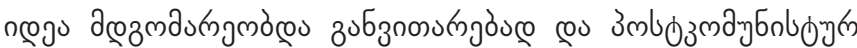

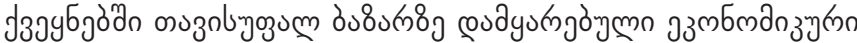

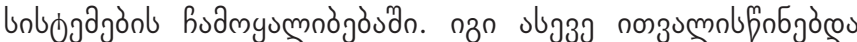

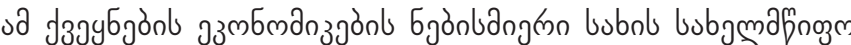

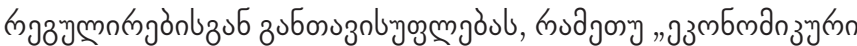

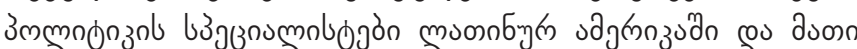

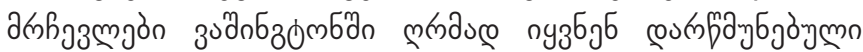

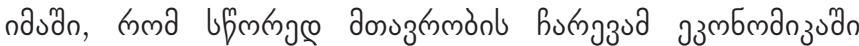

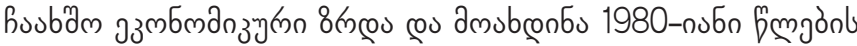

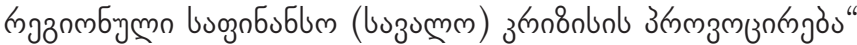
(Rodrik D. 2017).

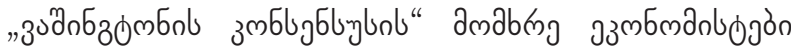

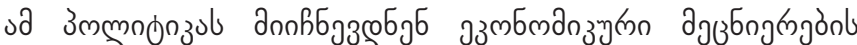

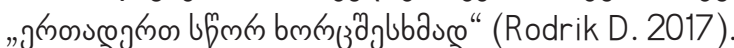

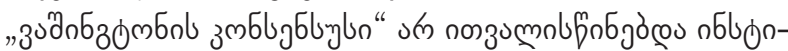

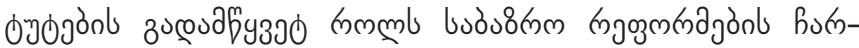

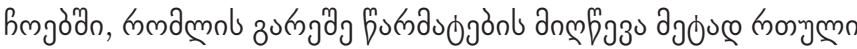

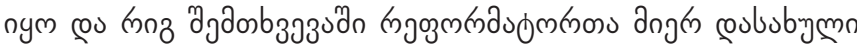

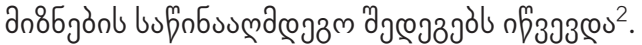

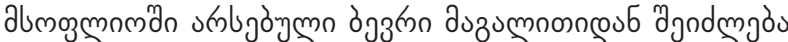

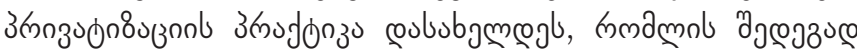

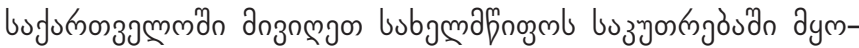

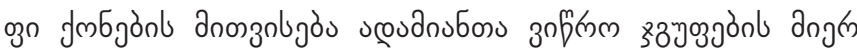

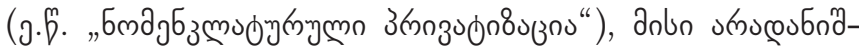

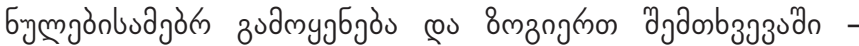

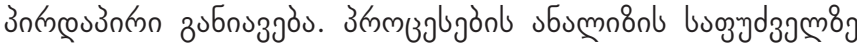

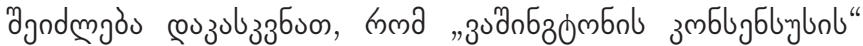

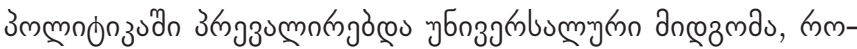

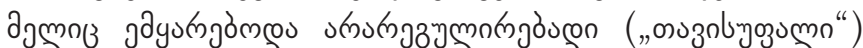

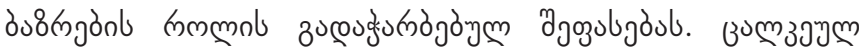

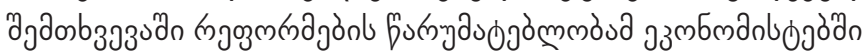

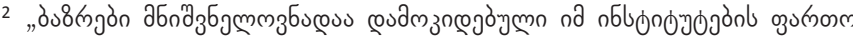

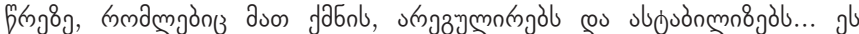

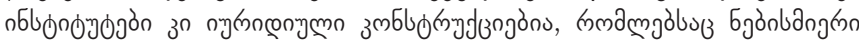

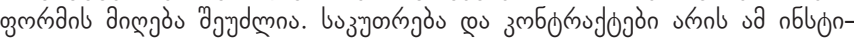

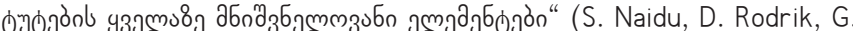
Zucman, 2019).
}

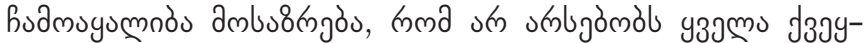

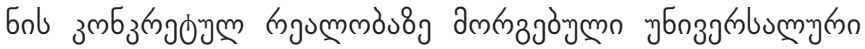

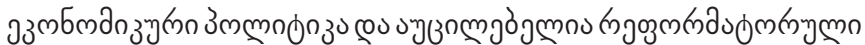

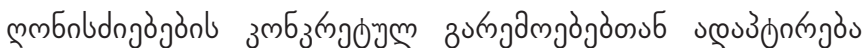
(Rodrik D. 2017).

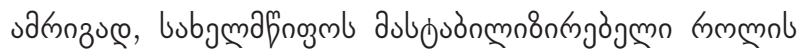

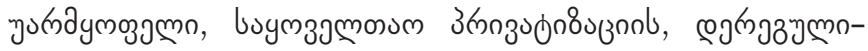

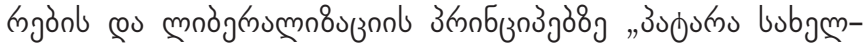

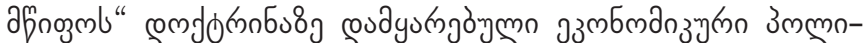

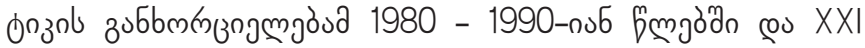

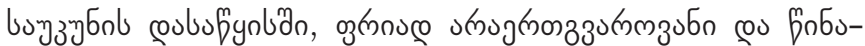

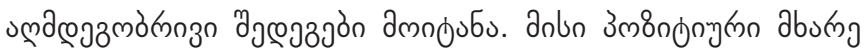

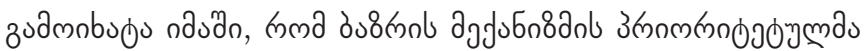

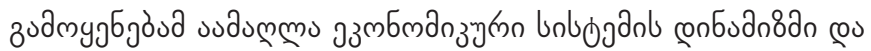

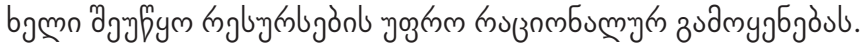

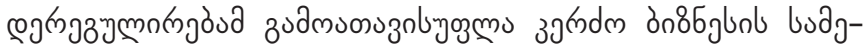

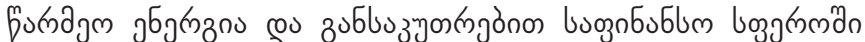

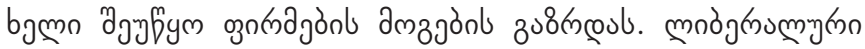

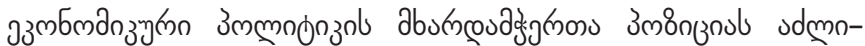

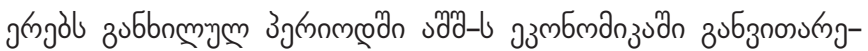

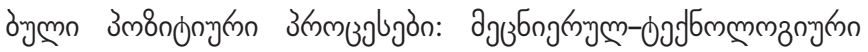

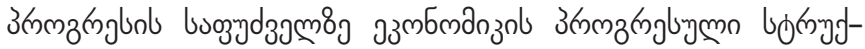
оy

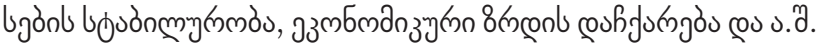

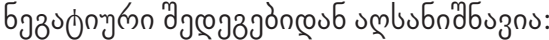

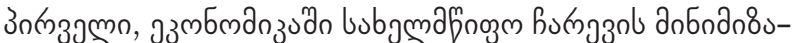

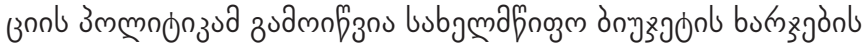

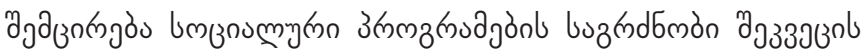
bumz8g, bymogul

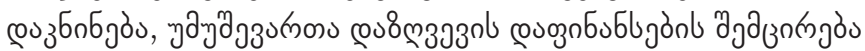

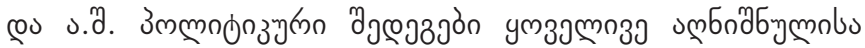

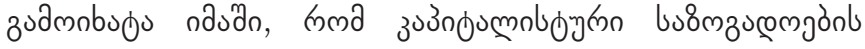

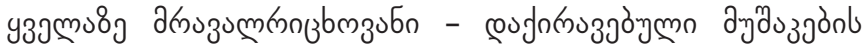

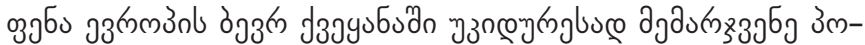

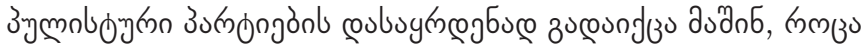

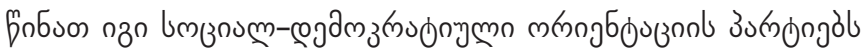

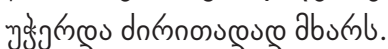

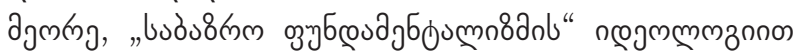

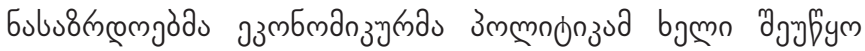

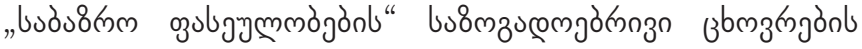

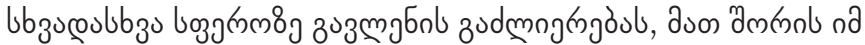

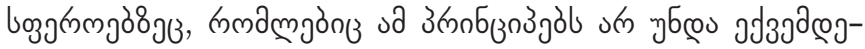

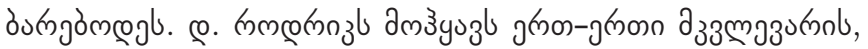

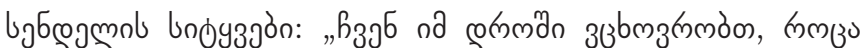

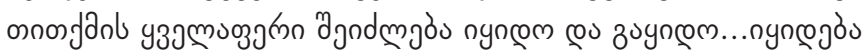
y3gmuoggrno" (Rodrik D. 2017). ${ }^{3}$

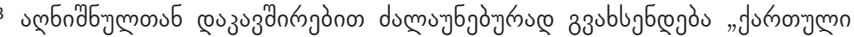

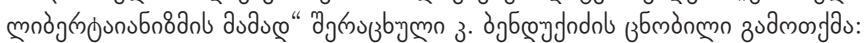




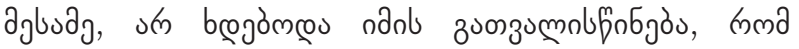

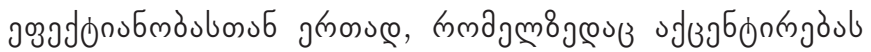

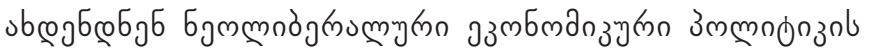

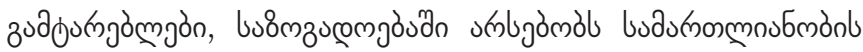

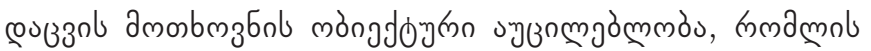

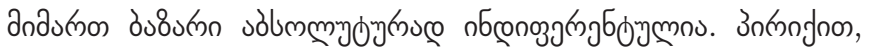

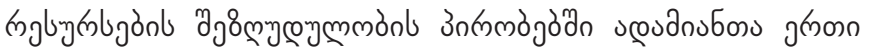

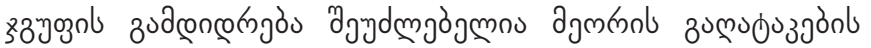

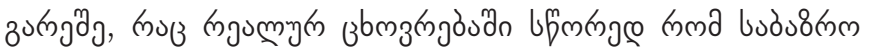

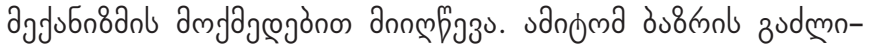

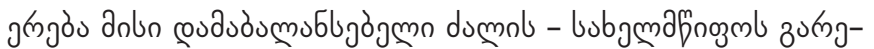

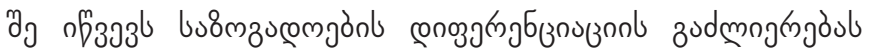

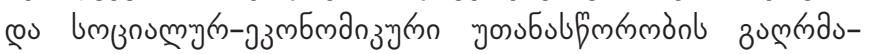

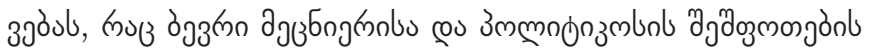
buzubnot.

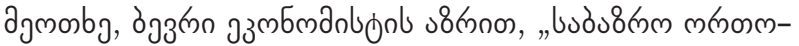

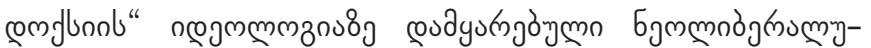

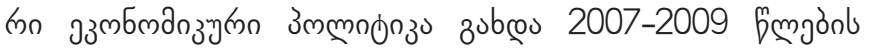

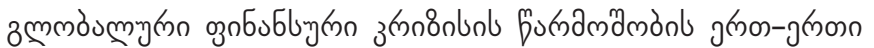

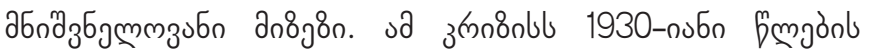

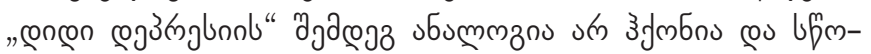

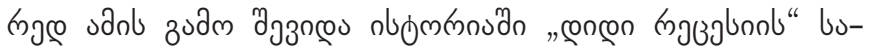

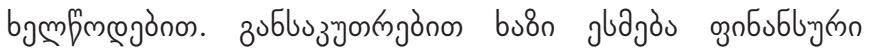

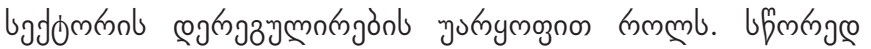

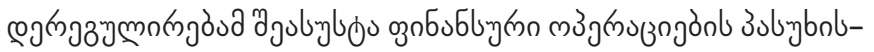
azjmm

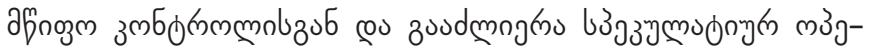

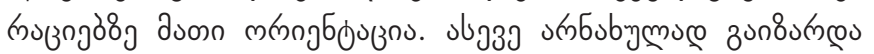

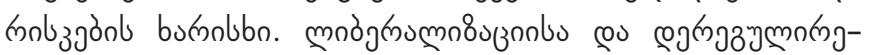

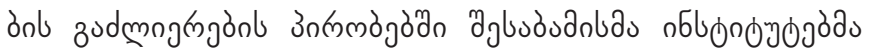
зэк

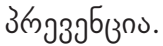

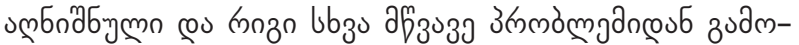

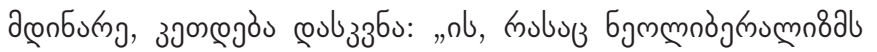

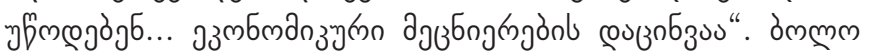

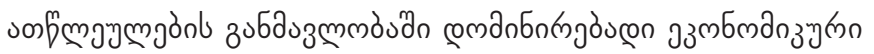

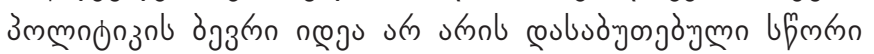

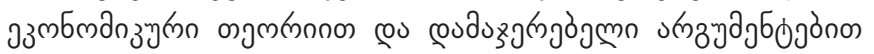
(S. Naidy, D. Rodrik, G. Zucman, 2019).

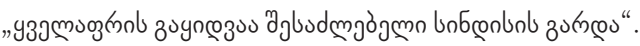

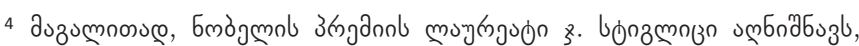

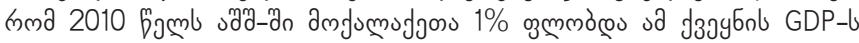

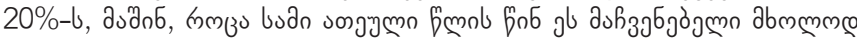

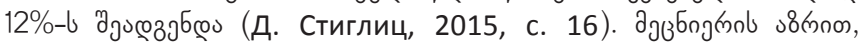

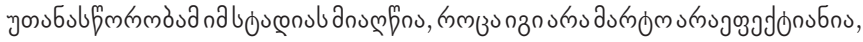

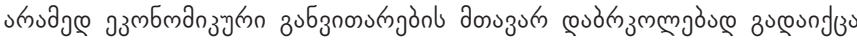
(Д. Стиглиц, 2015, с. 16). пзп Ј

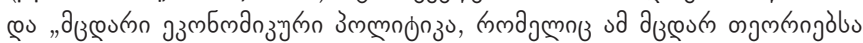

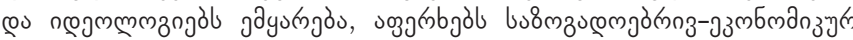

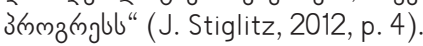

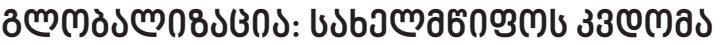

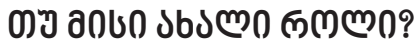

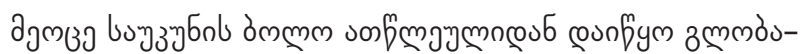
mo8uzznl nб

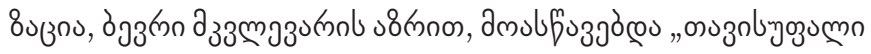

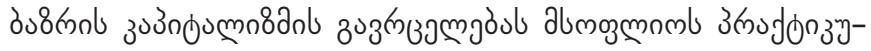
muœ yзgmo J3agubsdn" (Economic Report of the President. Washington, 2000, p.215; Nicholas V. Gianaris, 1996, p.

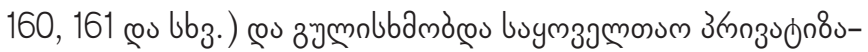

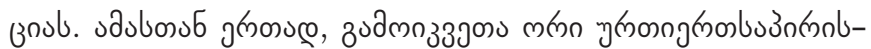

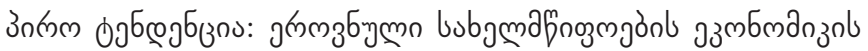

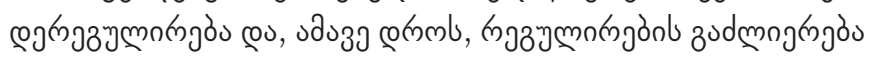

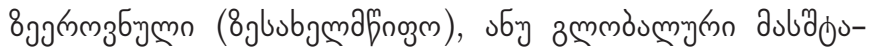

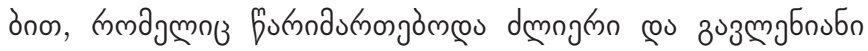

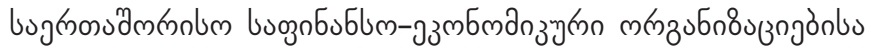

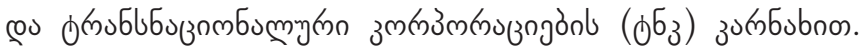

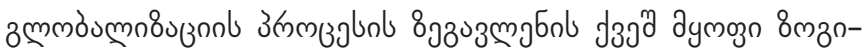

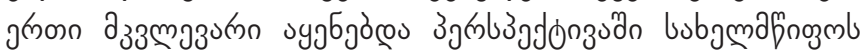

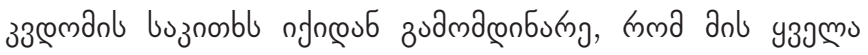

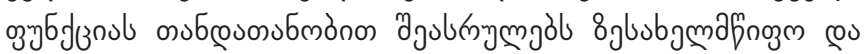

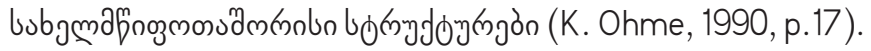

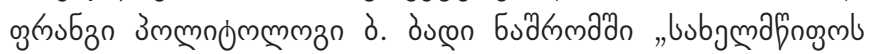

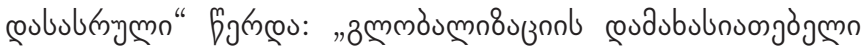

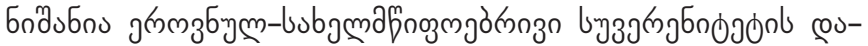

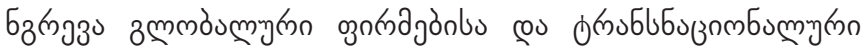

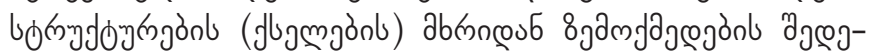
zง@्" (B. Badie, 1995, p. 12).

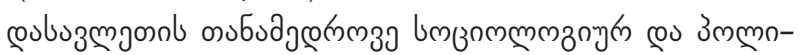
бмmмm

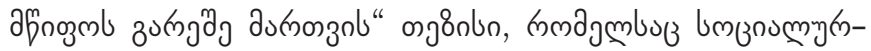

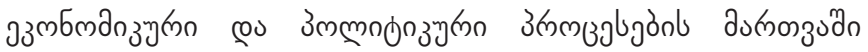

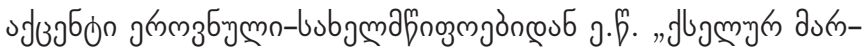
m308." zucouodzl (J. N. Rossenaw, 2003, w3, c.30 ).

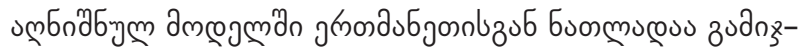

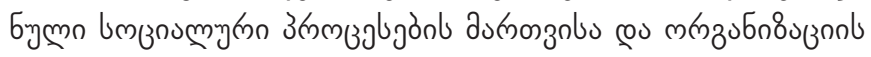

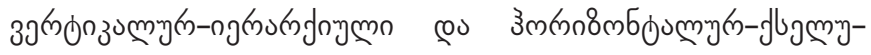

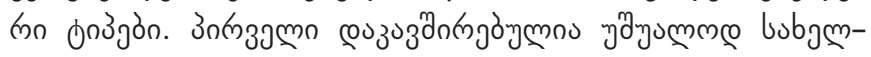

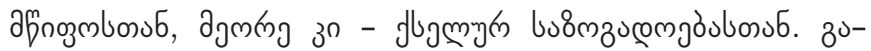

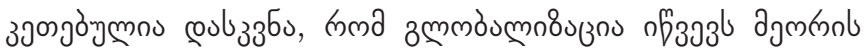

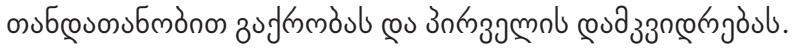

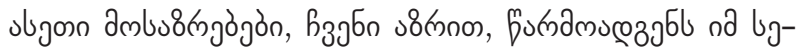

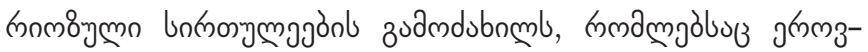

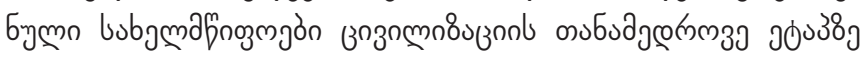

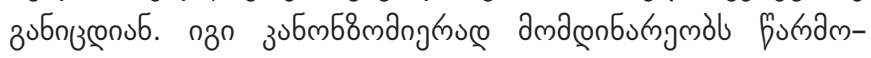

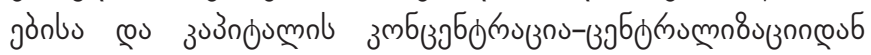
co ob

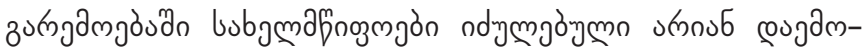

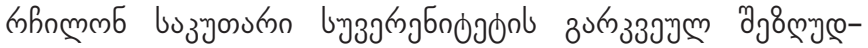

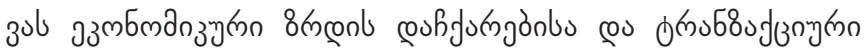




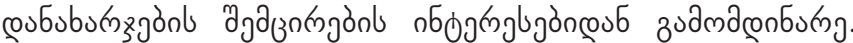

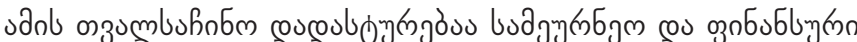

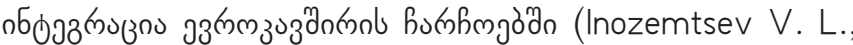
Kuznetsova E.S. 2002).

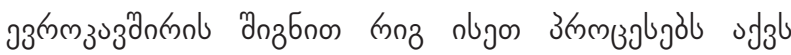

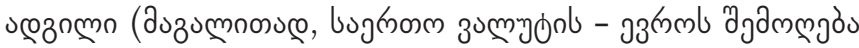

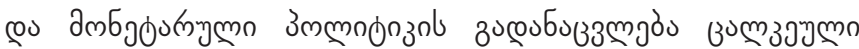

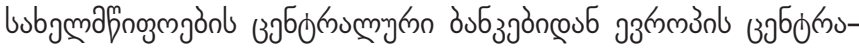

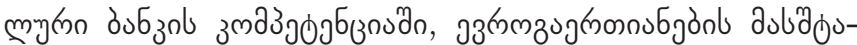

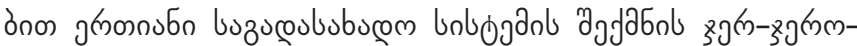

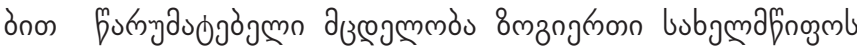

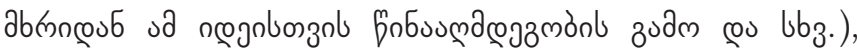

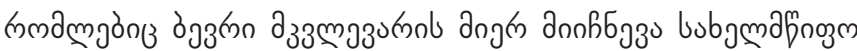

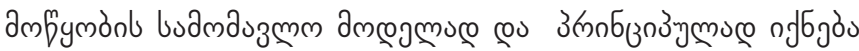

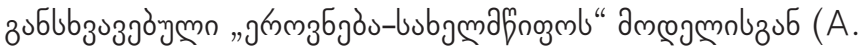
Linklater, 1998, p.200; M. Burgess, 2000, pp. 107-110, 140; T. Christiansen, K. Jougensen and A. Wiener, 2001, p. 14 cou bib3.)

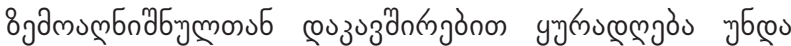

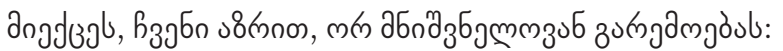

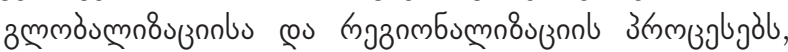

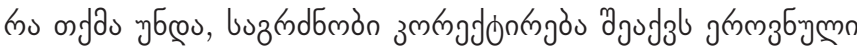

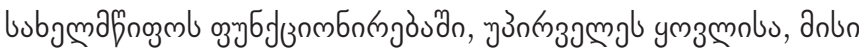

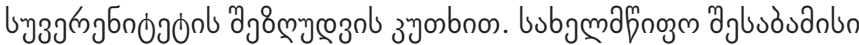

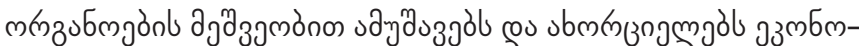

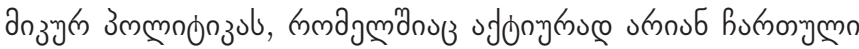

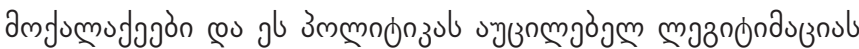
sकngुgal.

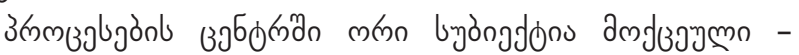

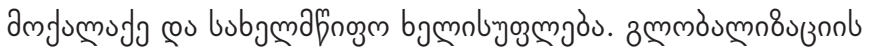

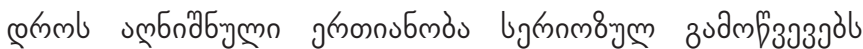

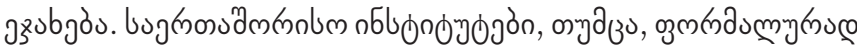

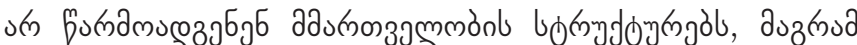

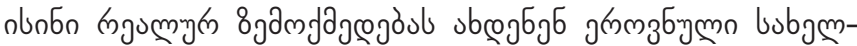

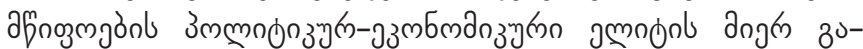

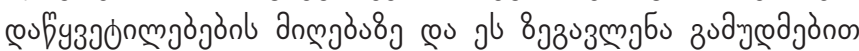

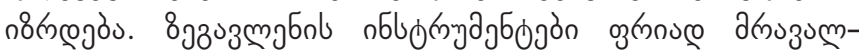

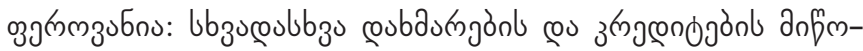

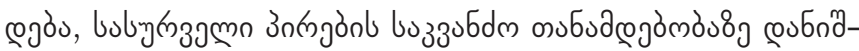
з

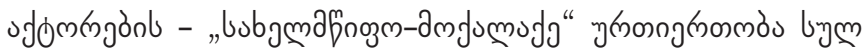

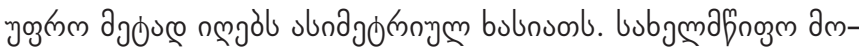

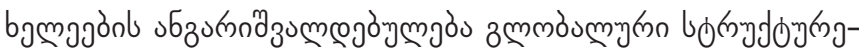

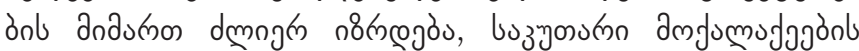

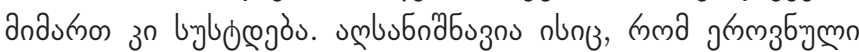

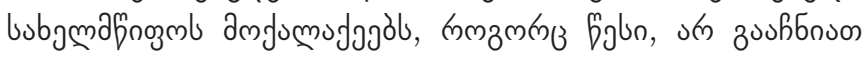

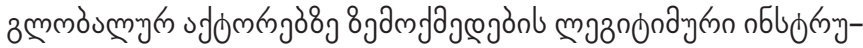

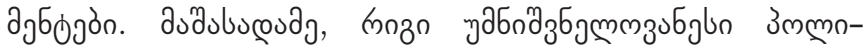

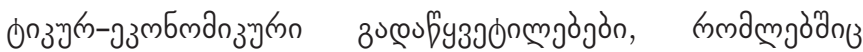

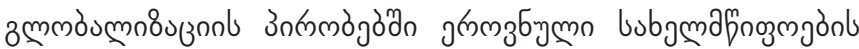

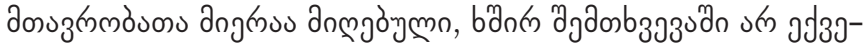

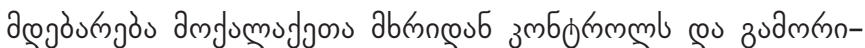
з зиз

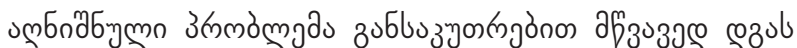

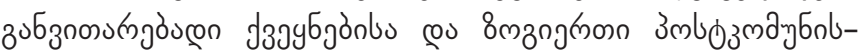

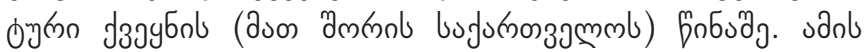

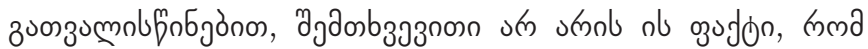

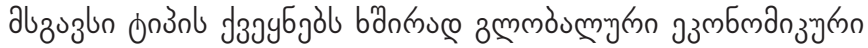

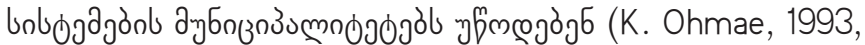
pp. 78-89).

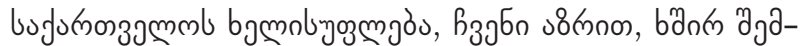

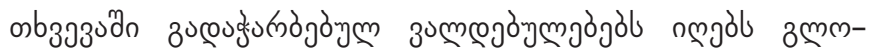

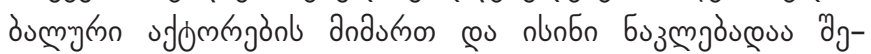

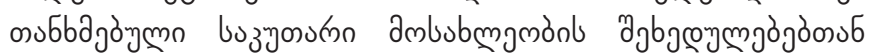

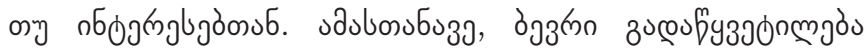

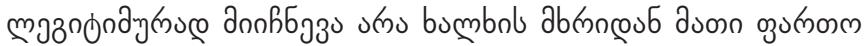

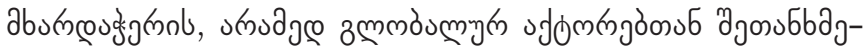
on zudm. s.

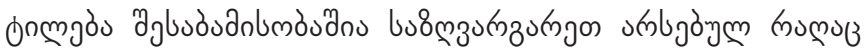

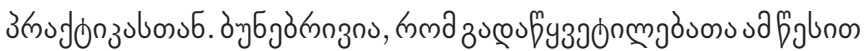

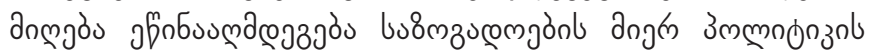

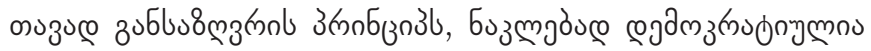

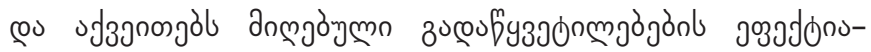

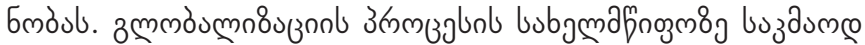

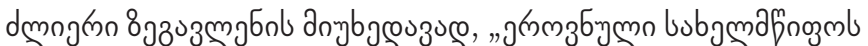

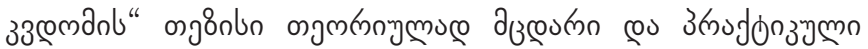

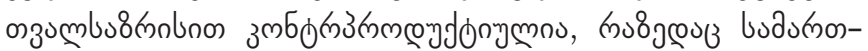

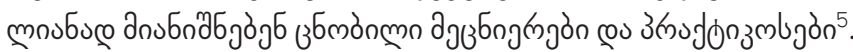

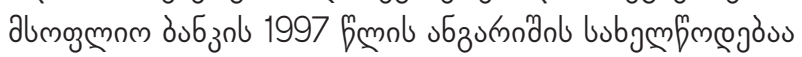

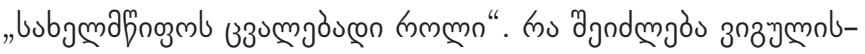

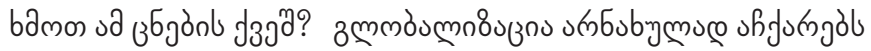

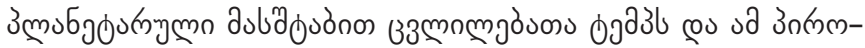

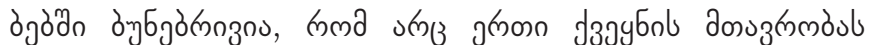

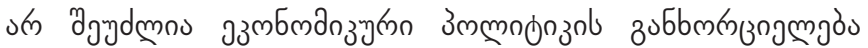

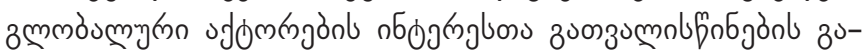

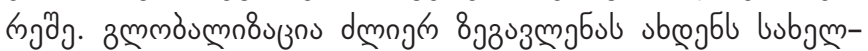

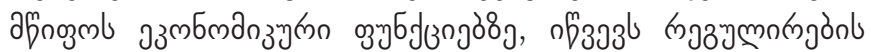

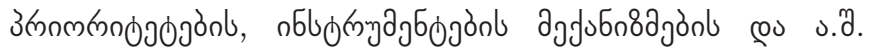

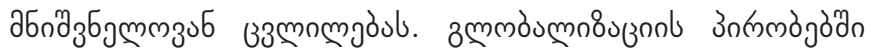

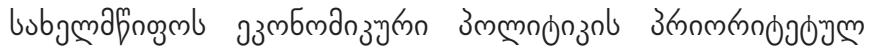

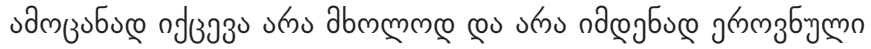

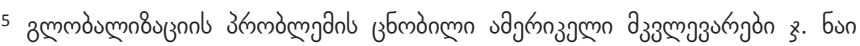

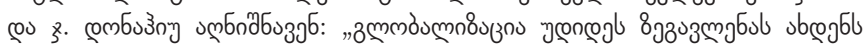

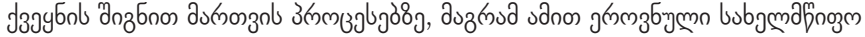

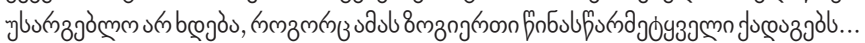

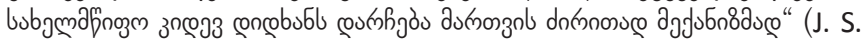

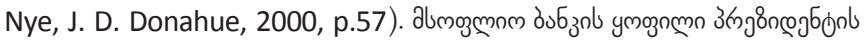

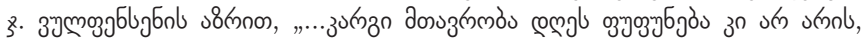

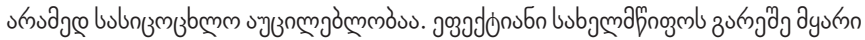

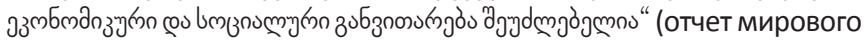
банка за 1997 год. М., 2002, с. 32 (оз bb3.).
} 


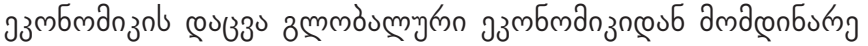

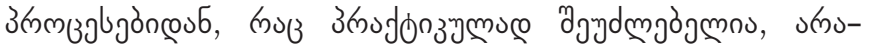

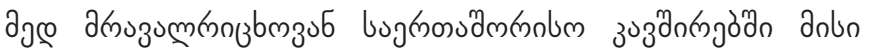

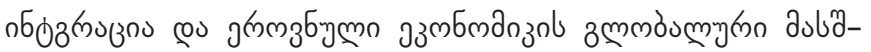

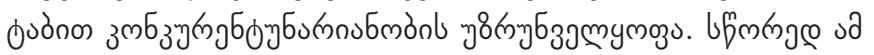
озимmosb

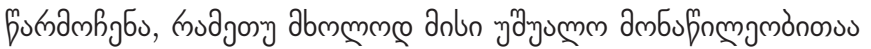

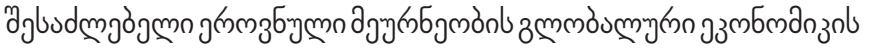

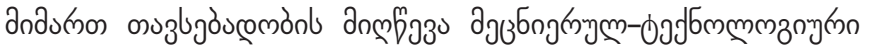

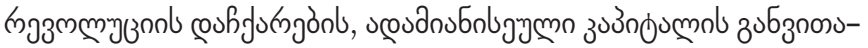

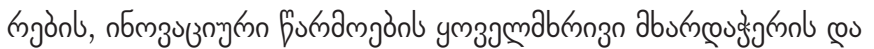
ง.ð. 38̊jònon (Mekvabishvili E. 2009).

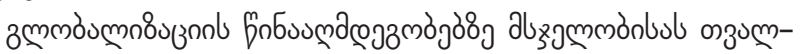

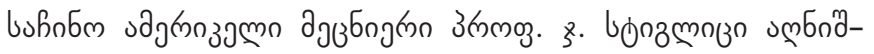
бuzb, mma n

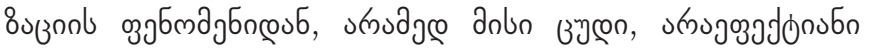

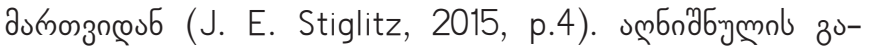

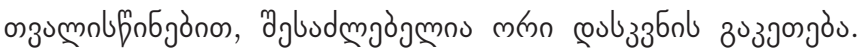

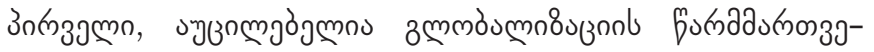

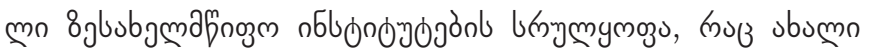

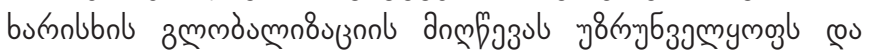

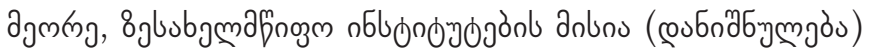

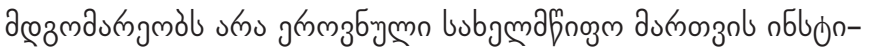

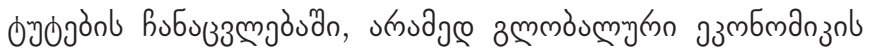

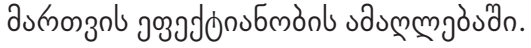

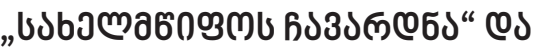

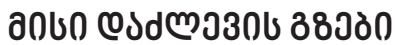

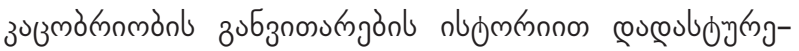

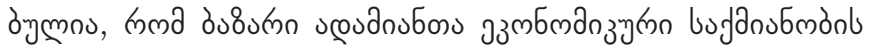

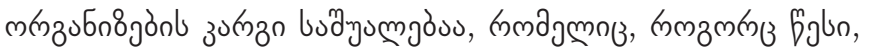

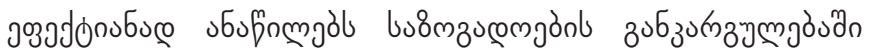

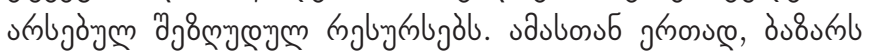

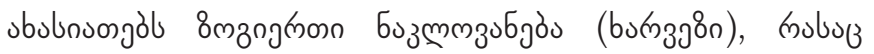

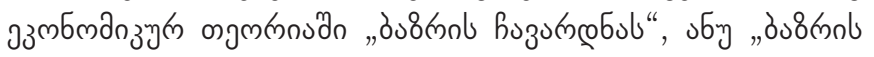

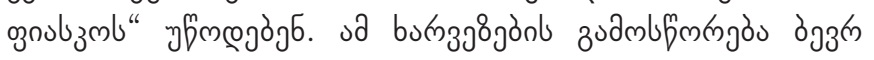

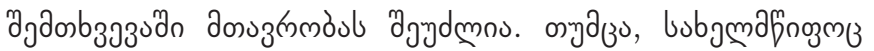

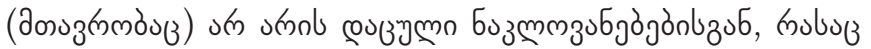

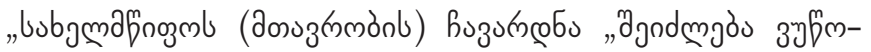

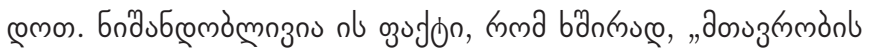

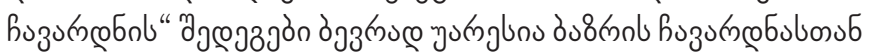

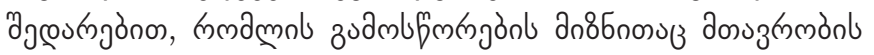

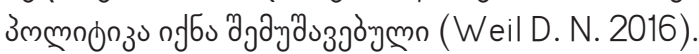

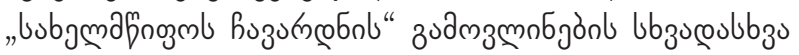

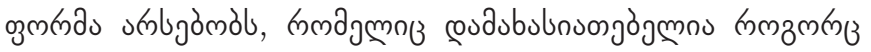

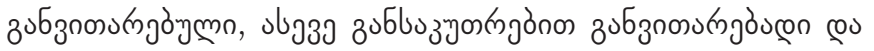

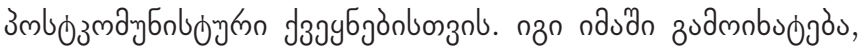

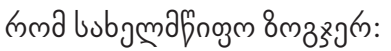

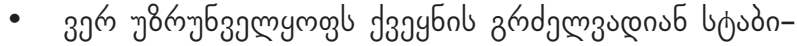

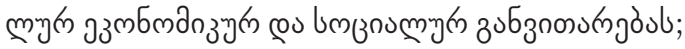

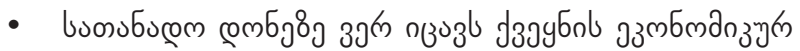

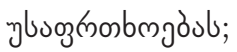

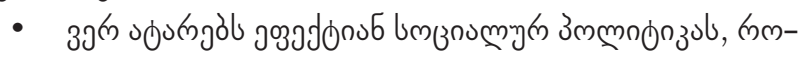

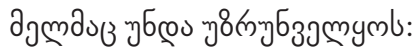

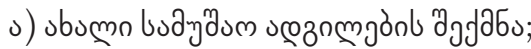

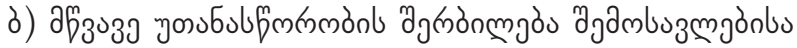

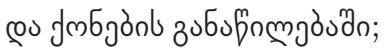

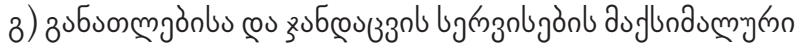

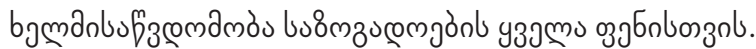

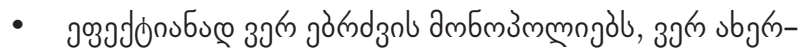

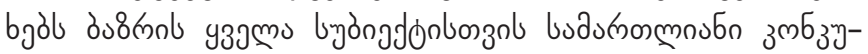

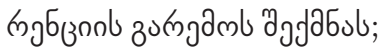

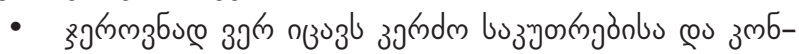

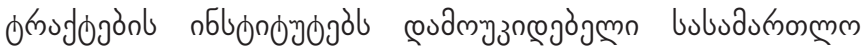

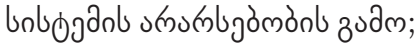

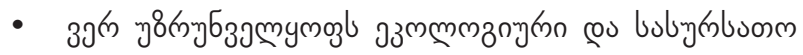

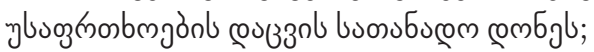

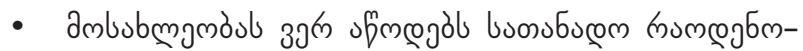

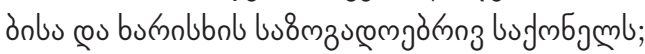

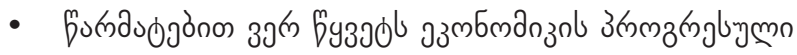

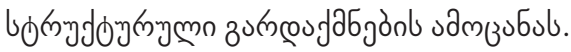

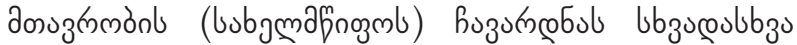

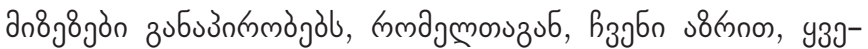

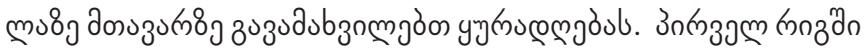

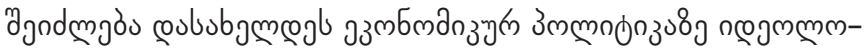

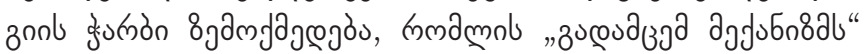
fumamuœ

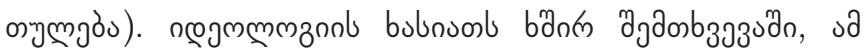

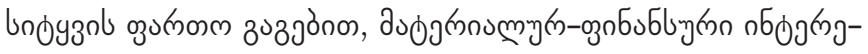
bgón zublusagzmozl.

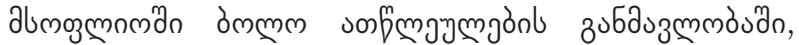

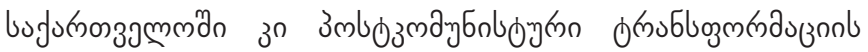

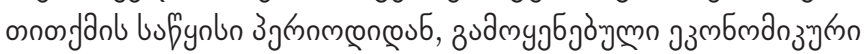

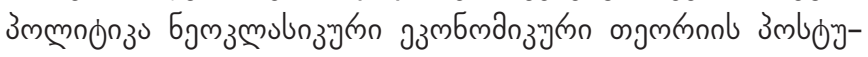

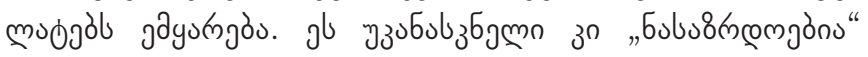

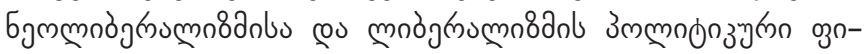

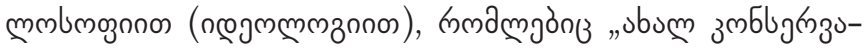

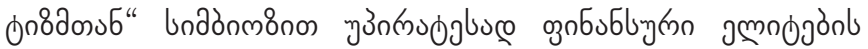

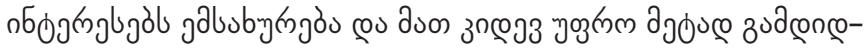

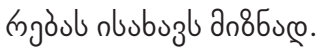

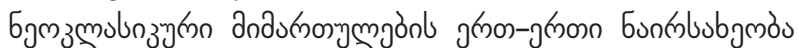

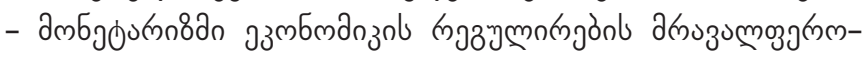

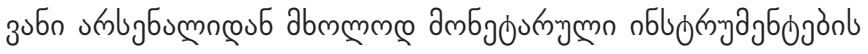

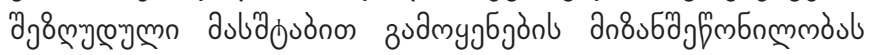

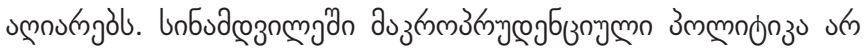

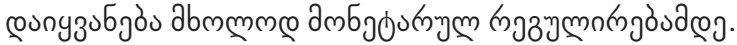

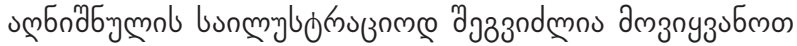

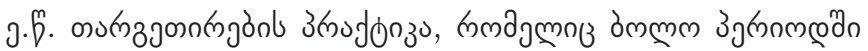




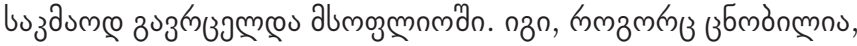

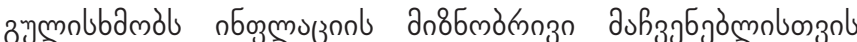

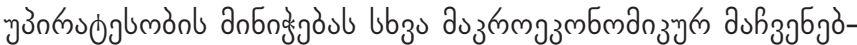

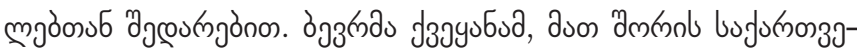

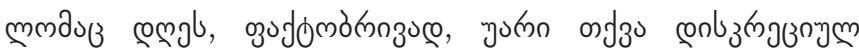

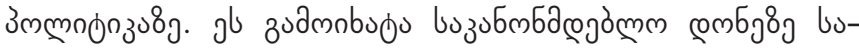

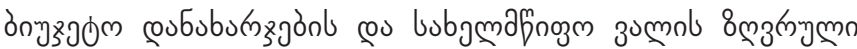

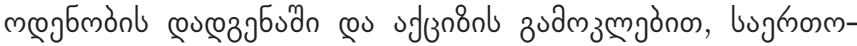

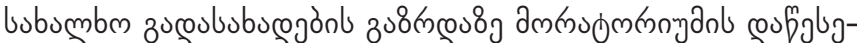

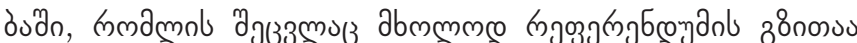

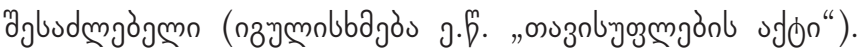

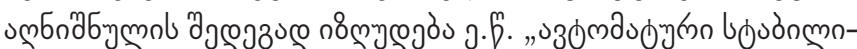

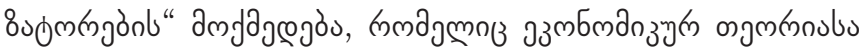

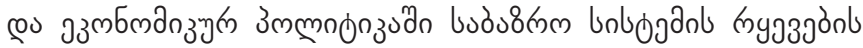

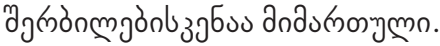

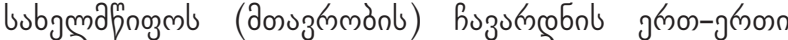

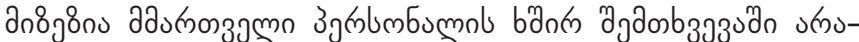

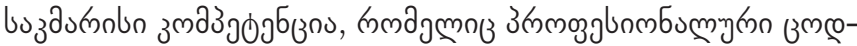

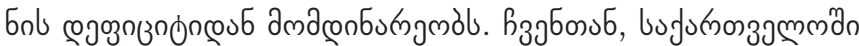

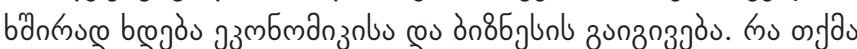

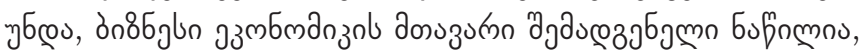
дn

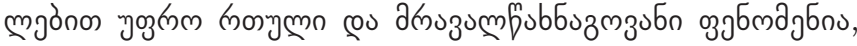

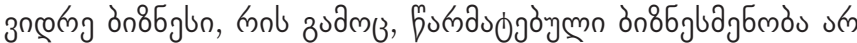

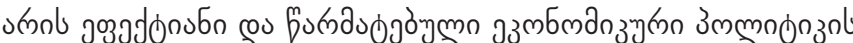

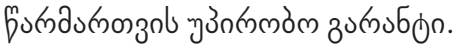

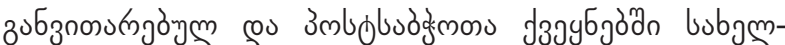

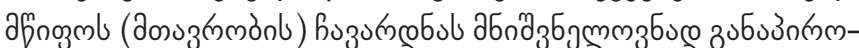

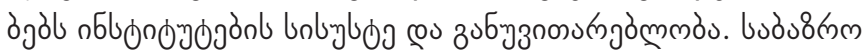

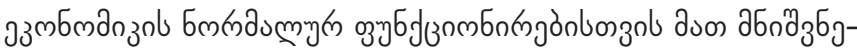

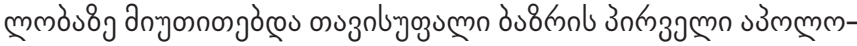

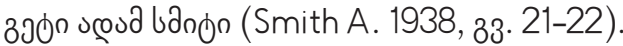

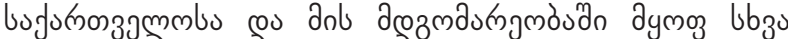

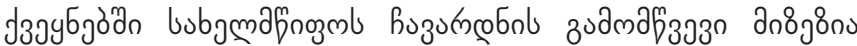

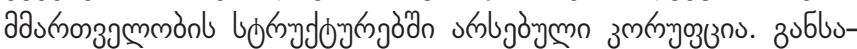

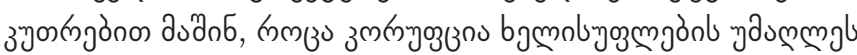

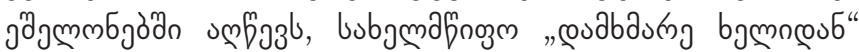

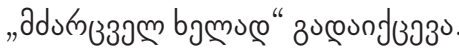

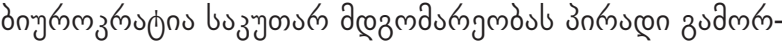

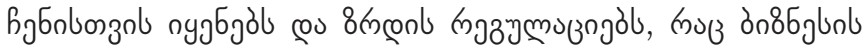

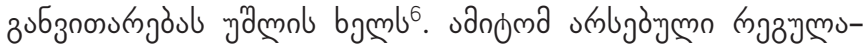

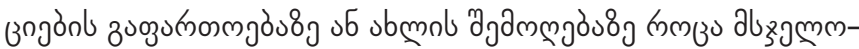

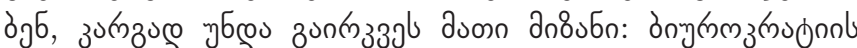
nб

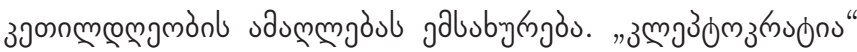

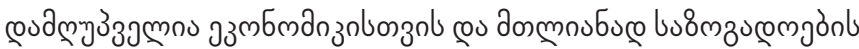

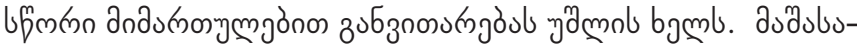

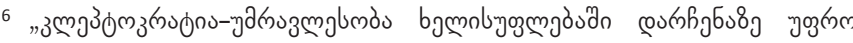

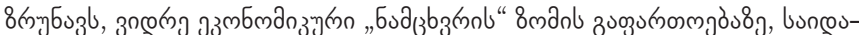

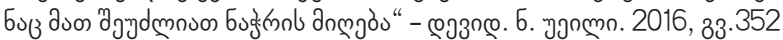

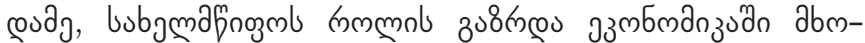

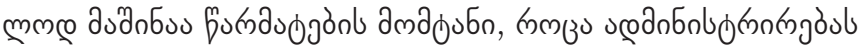

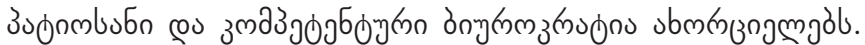

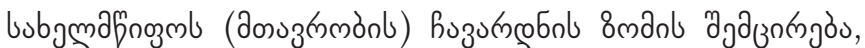

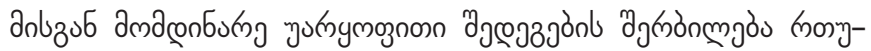

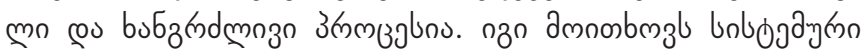

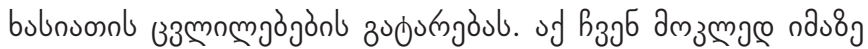

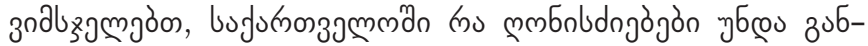

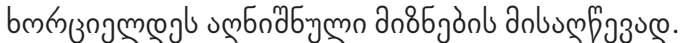

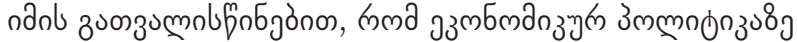

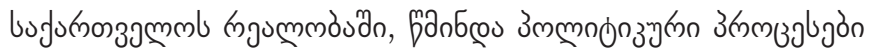

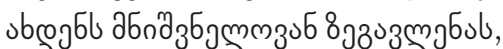

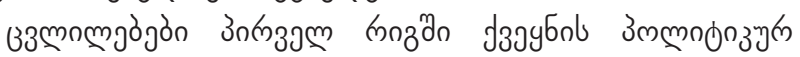

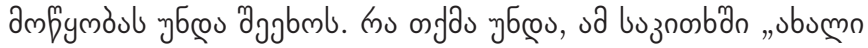

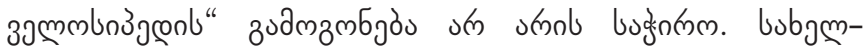

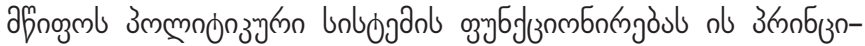

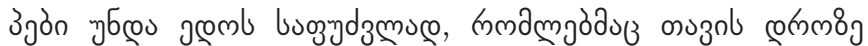

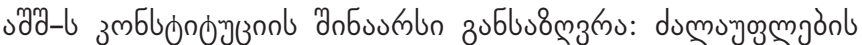

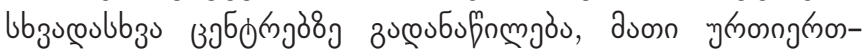

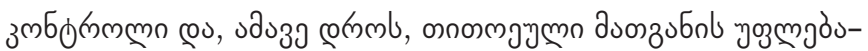

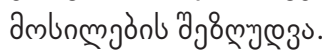

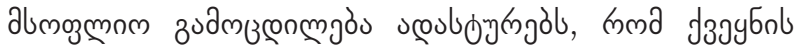

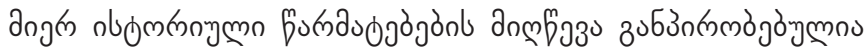
s

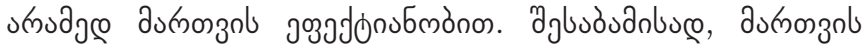

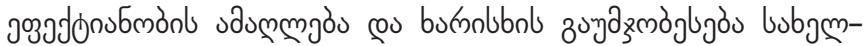

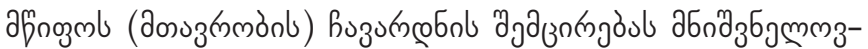

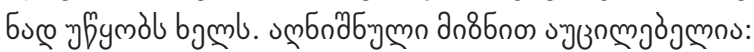

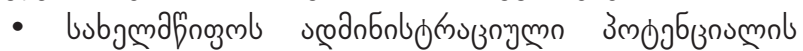

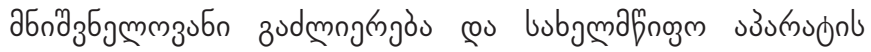
bufanobmònb bringmymoुs;

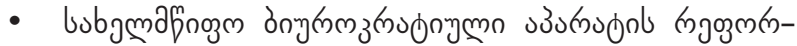

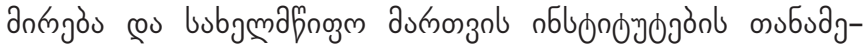

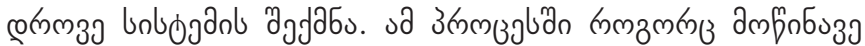

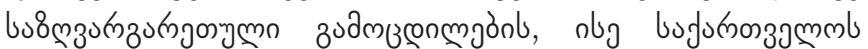

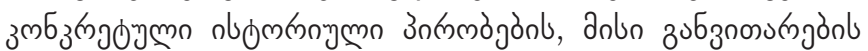

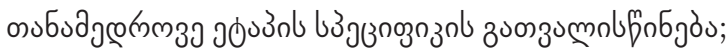

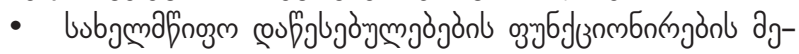

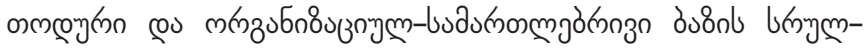
ymojs;

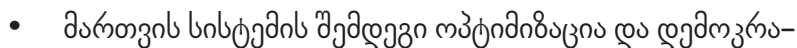
ono8\%3no;

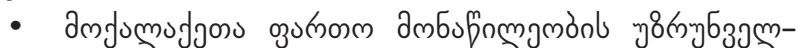

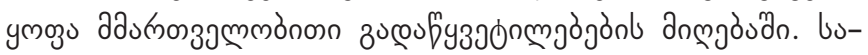

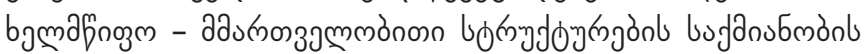

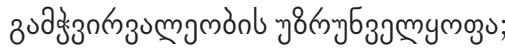

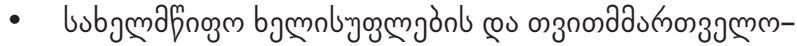

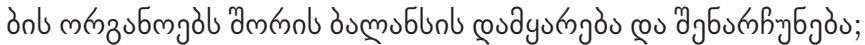

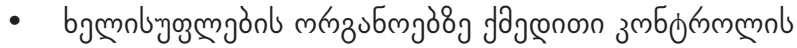

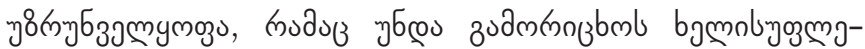




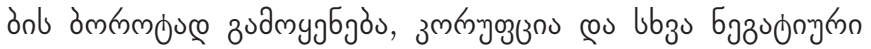
дmзмjлбjòn.

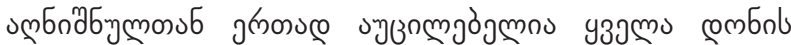

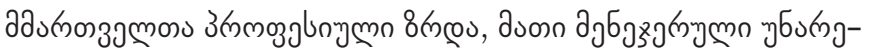

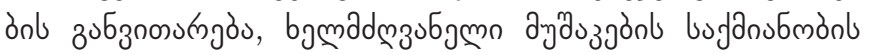

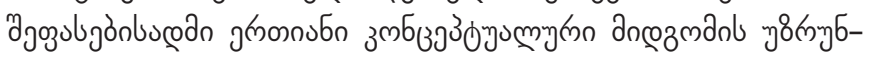

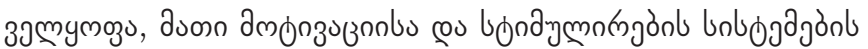

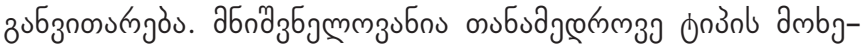

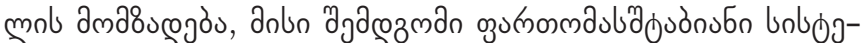

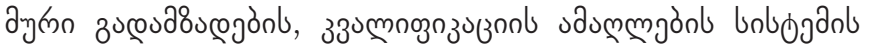

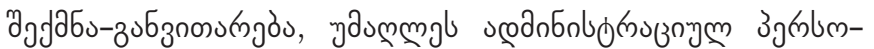

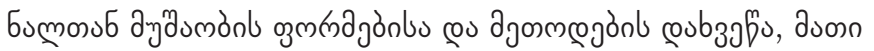

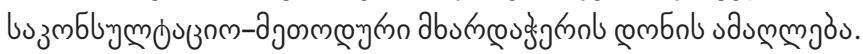

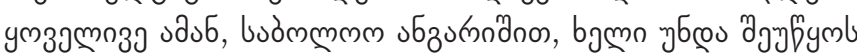

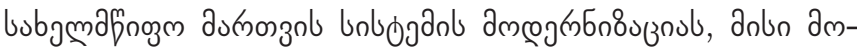

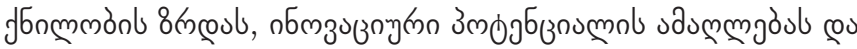

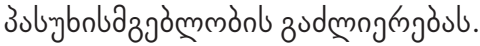

\section{@ob335s}

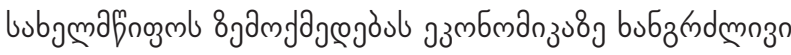

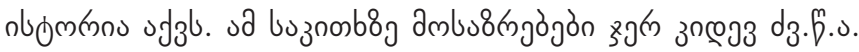

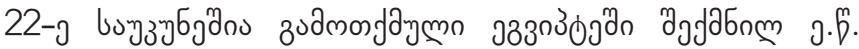

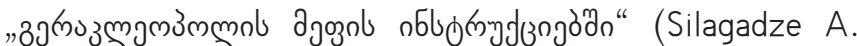

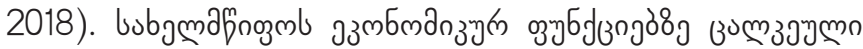

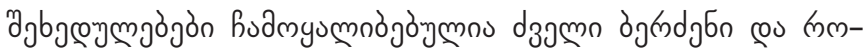

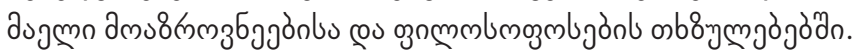

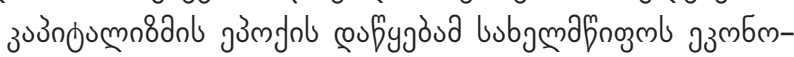

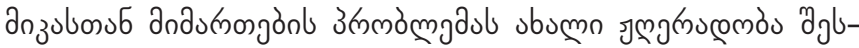

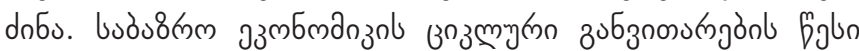

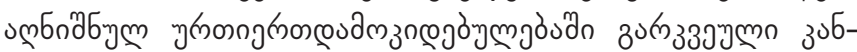

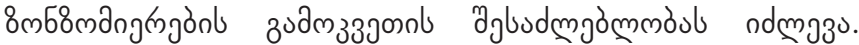

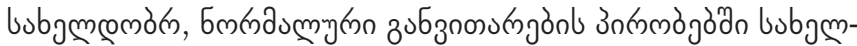

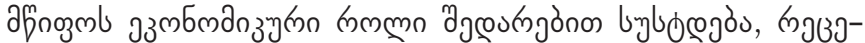

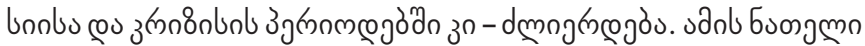

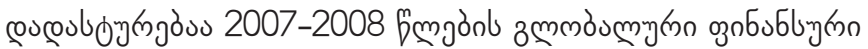

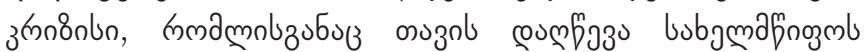
ujongran ubonz

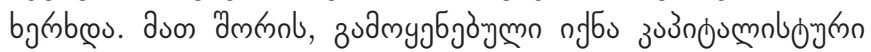

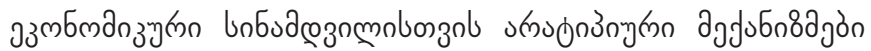

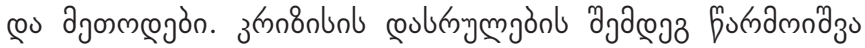

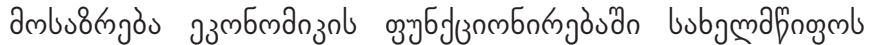

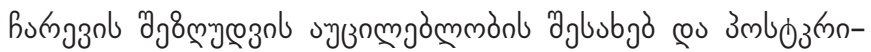

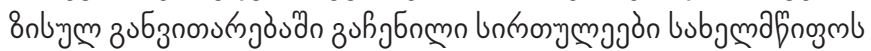
"0ud mumgou".

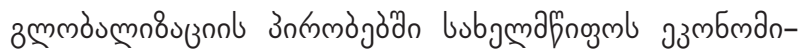

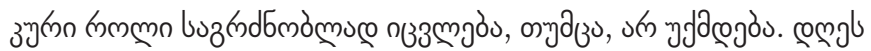

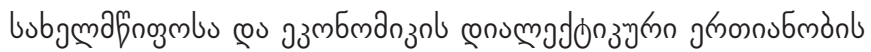
зuбalu8

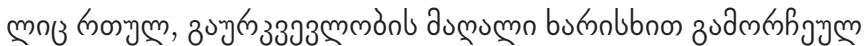

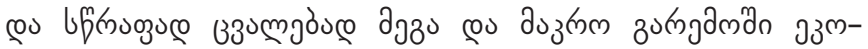

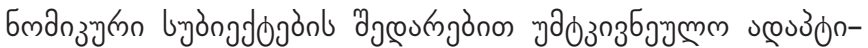

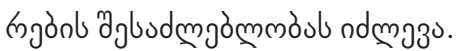

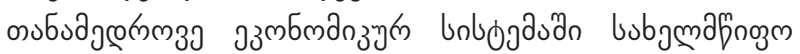

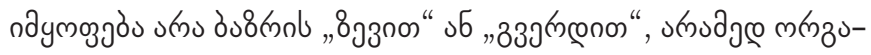

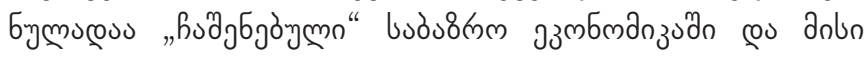

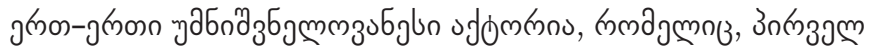

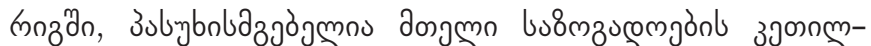

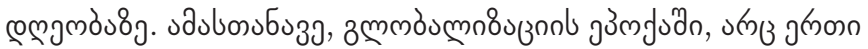

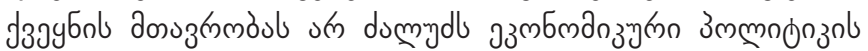

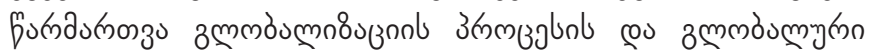

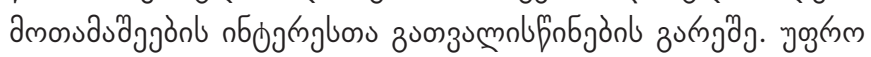

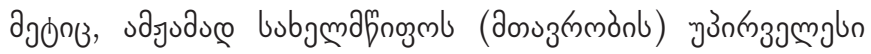

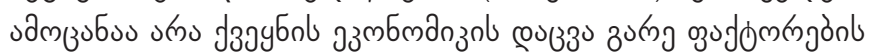

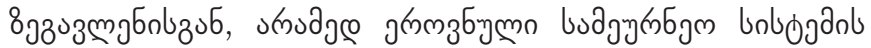

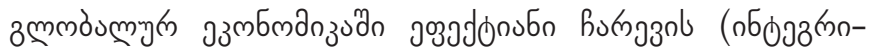

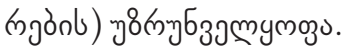

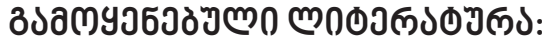

1. Asatiani R. (2010). Globalization, Economic Theory and Georgia. [Globalizatsia, Ekonomikuri Teoria da sakartvelo.] «News».

2. Aristotle (1995-1996). Politics: part I/II [Politika: natsili I/II], Tbilisi, 1995, 1996.

3. Blanchard O. (2010). Macroeconomics. [Makroekonomika.] Tbilisi University Press.

4. Castells, M. (2003). The scale of the game. magazine. “Economic strategies” №3.

5. Mankiw G. N. (2008). Principles of Economics. [Ekonomikis Printsipebi.] Publisher «Diogene».

6. Mekvabishvili E. (2009). Globalization of Economics: Directions, Challenges, Prospects. [Ekonomikis Globalizatsia: Mimartulebebi, Gamotsvevebi, Perspektivebi.] Publisher «Innovation».

7. Mekvabishvili e. (2018). The Financial Crisis of the Epoch of Globalization and the Economy of Georgia. [Globalizatsiis Epokis Finansuri Krizisebi da Sakartvelos Ekonomika.] Publisher «Intellect».

8. Roseanne H. S. (2006). Public Finances. [Sajaro Finansebi.]

9. Silagadze A. (2018). The Origins of Economic Science. [Ekonomikuri Metsnierebis Sataveebtan.] Tbilisi University Press.

10. Smith A. (1938). An Inquiry into the Nature and Causes of the Wealth of Nations. [Gamokvleva Khalkhta Simdidris Bunebisa da Mizezebis Shesakheb.] 
11. Weil D. N. (2016). Economic Growth. [Ekonomikuri Zrda.] Tbilisi University Press.

12. Hayek F. (2002). The Road to Serfdom. [Gza Monobisken.]

13. Badie B. (1995). La fin des territories.

14. Burgess M. (2000). Federalism and European Union: The Building of Europe, 1950-2000. London, New-York.

15. Christiansen T. Jougensen K. and Wiener A. (2001). The social construction of Europe. London.

16. Economic Report of the President (2000). Washington.

17. Gianaris N. V., (1996). Modern Capitalism: Privatization, Employee Ownership, and Industrial Democracy. Westport.

18. Hutton W. (2002). The World We're in. London: Little Brown.

19. Inozemtsev V. L., Kuznetsova E.S. (2002). The Return of Europe. Strokes to the portrait of the Old World in the new century. M. [Vazvrashenie Evropi. Shtrikhi k Portretu Starava Sveta v Novam Staletii. M]

20. Linklater A. (1998). The Transformation of Political Community: Ethical Foundations of the Post-Westphalian Era. Columbia (SC).

21. Meltzer A. H. (2005). Origins of the Great Inflation. Federal Reserve Bank of St. Louis Review.

22. Naidu S., Rodrik D., Zucman G. (2019). Economics After Neoliberalism. Boston Review, April 29, http://bostonreview.net/ forum/suresh-naidu-dani-rodrik-gabriel-zucman-economics-after-neoliberalism.

23. Nye J. S., Donahue J. D. (2000). Governance in a Globalizing World.

24. Ohmae K., (1990). The Borderless World: Power and Strategy in the Interlinked Economy.

25. Ohmae K., (1993). The Rise of Regional Economies.

26. Pierson Ch., (1995). Beyond the Welfare State? Cambridge.

27. Rosenau J. N., (1992). Governance without Government: Order and Change in World Politics. N. Y.

28. Rodrik D., (2016). The economy decides: the strength and weakness of the "dark science". [Ekonomika Rishaet: Sila $i$ Slabasts "Mrachnai Nauki".]

29. Stiglitz J. E., (2015). The Price of Inequality. [Cena Neravenstva.] N. Y.

30. Thurow L. C., (1981). The Zero-Sum Society: Distribution and the possibilities for economic change. London.

31. World Bank Report 1997. M., (1998). [Otchot Miravova Banka.]

32. Kastels M. (2003). Game Scale. [Mashtab Igri.] journal "Economic Strategy." 


\title{
THE ECONOMIC ROLE OF THE STATE IN CONDITIONS OF GLOBALIZATION
}

\author{
ELGUJA MEKVABISHVILI \\ Doctor of Economic Sciences, Professor, \\ Ivane Javakhishvili Tbilisi State University, \\ Academician of the Academy of \\ Economic Sciences of Georgia, Georgia \\ elguja.mekvabishvili@tsu.ge
}

For citation: Mekvabishvili, E. (2019). The Economic Role of the State in Conditions of Globalization, Globalization And Business, №8, pp. 22-33. https://doi.org/10.35945/gb.2019.08.002

\section{SUMMARY}

State (government) intervention in the economy has a long history. This issue has become especially crucial after the formation of the market economy. The cyclical (uneven) nature of market economy development determines state's intervention with various levels in certain periods. the role of government in economy is getting more important in recession and crisis periods and it is becoming less important in a period of the normal development of the economy. The most obvious proof of this is the global financial and economic crisis of 2007-2008, that has been overcome by the active anti-crisis policy of the State. The government's role in the economy during the post-crisis period was relatively weakened. Globalization significantly changes the state's economic role, but it does not nullify it. Nowadays, the relationship between the state and the economy is crucial and beneficial for both sides.

In the modern economic system the state has one of the most important parts of the market economy. Unlike the private market, which is determined by the profit and-loss categories, the main objective of the Statess economic activity is to maximize welfare of the entire society. Governments of the countries participating in the globalization process are not able to carry out economic policy without taking into consideration the interests of the global members - international financial and economic institutions, transnational companies (TNCS) and regional unions. 